\title{
Leakage-resilient non-malleable codes
}

\author{
Divesh Aggarwal $^{*} \quad$ Stefan Dziembowski $^{\dagger} \quad$ Tomasz Kazana $^{\ddagger} \quad$ Maciej Obremski $^{\S}$
}

September 18, 2015

\begin{abstract}
A recent trend in cryptography is to construct cryptosystems that are secure against physical attacks. Such attacks are usually divided into two classes: the leakage attacks in which the adversary obtains some information about the internal state of the machine, and the tampering attacks where the adversary can modify this state. One of the popular tools used to provide tamper-resistance are the non-malleable codes introduced by Dziembowski, Pietrzak and Wichs (ICS 2010). These codes can be defined in several variants, but arguably the most natural of them are the information-theoretically secure codes in the $k$-split-state model (the most desired case being $k=2$ ).

Such codes were constucted recently by Aggarwal et al. (STOC 2014). Unfortunately, unlike the earlier, computationally-secure constructions (Liu and Lysyanskaya, CRYPTO 2012) these codes are not known to be resilient to leakage. This is unsatisfactory, since in practice one always aims at providing resilience against both leakage and tampering (especially considering tampering without leakage is problematic, since the leakage attacks are usually much easier to perform than the tampering attacks).

In this paper we close this gap by showing a non-malleable code in the 2-split state model that is secure against leaking almost a 1/12-th fraction of the bits from the codeword (in the bounded-leakage model). This is achieved via a generic transformation that takes as input any non-malleable code (Enc, Dec) in the 2-split state model, and constructs out of it another nonmalleable code $\left(\mathrm{Enc}^{\prime}, \mathrm{Dec}^{\prime}\right)$ in the 2-split state model that is additionally leakage-resilient. The rate of $\left(E^{\prime} c^{\prime}, \mathrm{Dec}^{\prime}\right)$ is linear in the rate of $(\mathrm{Enc}, \mathrm{Dec})$. Our construction requires that $\mathrm{Dec}$ is symmetric, i.e., for all $x, y$, it is the case that $\operatorname{Dec}(x, y)=\operatorname{Dec}(y, x)$, but this property holds for all currently known information-theoretically secure codes in the 2-split state model. In particular, we can apply our transformation to the code of Aggarwal et al., obtaining the first leakage-resilient code secure in the split-state model. Our transformation can be applied to other codes (in particular it can also be applied to a recent code of Aggarwal, Dodis, Kazana and Obremski constructed in the work subsequent to this one).
\end{abstract}

\footnotetext{
${ }^{*}$ Department of Computer and Communication Sciences EPFL. Email: Divesh.Aggarwal@epfl.ch.

${ }^{\dagger}$ Institute of Informatics, University of Warsaw. Email: std@mimuw.edu.pl.

${ }^{\ddagger}$ Institute of Informatics, University of Warsaw. Email: tkazana@mimuw.edu.pl.

${ }^{\S}$ Institute of Informatics, University of Warsaw. Email: obremski@mimuw.edu.pl.
} 


\section{Introduction}

Several attacks on cryptographic devices are based on exploiting physical weaknesses in their implementations. Such "physical attacks" are usually based on the side-channel information about the internals of the cryptographic device that the adversary can obtain by measuring its running-time, electromagnetic radiation, power consumption (see e.g. [ECR]), or on active tampering (see e.g. [AK96, ECR]). A recent trend in theoretical cryptography, initiated by [MR04, ISW03, IPSW06], is to design schemes that are provably-secure even if their implementations can be attacked.

One of the tools used in this area are the so-called non-malleable codes introduced by Dziembowski, Pietrzak and Wichs in 2010 [DPW10]. Informally, a code $($ Enc $: \mathcal{M} \rightarrow \mathcal{C}$, Dec $: \mathcal{C} \rightarrow \mathcal{M})$, where Enc is a randomized encoding function, and Dec is a partial decoding function, is nonmalleable if an adversary that learns $C=\operatorname{Enc}(M)$ is not able to produce $C^{\prime}=h(C)$ such that $\operatorname{Dec}\left(C^{\prime}\right)$ is not equal to $M$, but is "related" to it. The precise meaning of "not being related" is a little tricky to define but intuitively, what we require is that $C^{\prime}$ does not depend on $C$ in any non-trivial way. For example: $C^{\prime}$ equal to $C$ with the first bit set to zero, or $C^{\prime}$ equal to $C$ with every bit negated, are obviously "related" to $C$, but a uniformly random $C^{\prime}$, or a constant $C^{\prime}$ are unrelated to $C$. It is easy to see that in order to construct such codes, one needs to restrict in some way the set of possible "manipulation functions" $h$ that the adversary can use in order to compute $C^{\prime}$ from $C$. This is because otherwise the adversary could simply let $h$ compute $M$ from $C$ (using the decoding function Dec), compute $M^{\prime}$ that is "related to $M^{\prime}$ " (by say, negating all the bits of $M)$, and then output $C^{\prime}=\operatorname{Enc}\left(M^{\prime}\right)$. Therefore the non-malleable codes are always defined with respect to a family $\mathcal{H}$ of manipulation functions $h$ that the adversary is allowed to use to compute $C^{\prime}$ from $C$.

The main application for this notion is the protection against tampering attacks. Imagine $C=\operatorname{Enc}(M)$ is stored on some device that the adversary can tamper with, and hence he can substitute $C$ with some $C^{\prime} \neq C$. Suppose (Enc, Dec) is non-malleable with respect to the set of manipulation functions that the adversary is able to induce by tampering. Then the only thing that the adversary can achieve is that $C^{\prime}$ will either decode to the same $M$, or to some $M^{\prime}$ that is totally unrelated to $M$. This is useful, since many practical attacks on cryptographic schemes are based on the so-called "related key attacks" [BK03], where the adversary is able to break a scheme $\mathrm{S}(K)$ (where $K$ is the secret key) by having access to a device $\mathrm{S}\left(K^{\prime}\right)$ for some $K^{\prime}$ that is related to $K$. Clearly, storing $K$ in an encoded form $\operatorname{Enc}(K)$ provides protection against such attacks. In [DPW10] the authors describe also other applications of non-malleable codes. In particular they show how to use them in combination with the algorithmic tamper proof security framework of Gennaro et al. $\mathrm{GLM}^{+} 04$. Recently, Faust et al. used the non-malleable codes to construct Random Access Machines secure against tampering and leakage attacks [FMNV14b], and Coretti et al. CMTV14] have shown how to use the non-malleable codes to construct public-key encryption schemes.

Since the invention of the non-malleable codes, there has been a significant effort to construct codes that would be secure against interesting classes of families. In [DPW10] the authors show a construction of efficient codes secure against bit-wise tapering, i.e. when every bit of the codeword is manipulated independently (this is achieved using the algebraic manipulation detection codes of Cramer et al. [CDF $\left.\left.{ }^{+} 08\right]\right)$. They also provide an existential result that for every sufficiently small family $\mathcal{H}$ of manipulation functions there exists a (not necessarily efficient) non-malleable code secure against it. This immediately gives a construction of non-malleable codes secure in the random oracle model BR93. 
Previous constructions of non-malleable codes in the split-state model. A very attractive and natural family of manipulation functions can be defined using the so-called split-state model. Assume that $C$ is represented as a sequence of blocks $C=\left(C_{1}, \cdots, C_{k}\right)$. Then $\mathcal{H}$ is a family of $k$-split state manipulation functions if every $h \in \mathcal{H}$ manipulates each element $C_{1}, \ldots, C_{k}$ independently, i.e., for every $h$ there exist functions $\left\{h_{i}\right\}_{i=1}^{k}$ such that $h\left(C_{1}, \ldots, C_{k}\right)=\left(h_{1}\left(C_{1}\right), \ldots, h_{k}\left(C_{k}\right)\right)$. A practical justification for such a model comes from an observation that it may be easy to achieve in real life, by simply placing every $C_{i}$ on a separate chip. Of course, the fewer parts are needed, the stronger the model is, and in particular the most desirable case is $k=2$. In the sequel, we will sometimes refer to the 2-split state model simply as the "split model".

The aforementioned existential result of [DPW10] implies that there exist non-malleable codes in the 2-split state model. The problem of showing an efficient construction of such codes was left open in [DPW10]. The first step towards solving it was made by Liu and Lysyanskaya [LL12], who showed a construction of non-malleable codes computationally secure in the 2-split state model. Dziembowski, Kazana and Obremski [DKO13] provided an efficient construction of informationtheoretically non-malleable codes that works only for messages of length 1 . The problem of constructing information-theoretically secure codes for messages of arbitrary length was finally solved by Aggarwal, Dodis and Lovett [ADL14]. Their construction is based on the methods from additive combinatorics, including the so-called Quasi-polynomial Freiman-Ruzsa Theorem, and involves a substantial blow-up in the size of the codeword $\left(|C|=\tilde{O}\left((|M|+\kappa)^{7}\right)\right.$, where $\kappa$ is the security parameter). Very recently Chattopadhyay and Zuckerman [CZ14 have shown a construction of non-malleable codes in 10-split state model that achieves linear blow-up.

A subsequent construction of non-malleable codes in the split-state model. In a paper subsequent to this one, Aggarwal et al. [ADKO14] show a general transformation of any $k$-split state model secure non-malleable code into one secure in the 2 -split state model, that involves a linear blow-up in the codeword size. This, together with the result of [CZ14] gives a construction of a non-malleable code in the 2-split state model with codeword of length linear in $|M|$. Their construction uses some of the techniques developed in this work. In particular, one of the steps of their construction which can be seen as a generalization of our reduction is a reduction from the 2-split-state tampering family to the so called 2-part $t$-lookahead tampering family. However, the result of ADKO14 does not consider leakage-resilience, and considering the number of levels of encoding required by their result, it is unlikely that their construction is resilient to any significant leakage.

Leakage-resilience of non-malleable codes. The ultimate goal of the "physically secure cryptography" is to provide both tampering- and leakage-resilience. The basic definition of the nonmalleable codes does not consider any type of leakage information that the adversary can obtain about the codeword through the side channels. This may be considered unrealistic, as in practice it may be often relatively easy for the adversary to obtain such information (probably easier than to perform the tampering attacks). Therefore, it would be desirable to include also such attacks in this definition. The first paper that considered leakage-resilient non-malleable codes was [LL12] (in the computational settings). Our definition essentially follows their ideas, except that we consider the information-theoretic settings.

Let us now explain informally the concept of leakage-resilient non-malleable codes in the 2 -split state model (the formal definition appears in Section 3). First consider the question what would be the most natural definition of leakage and tampering attacks. To be as general as possible we should give to the adversary right to simultaneously tamper the codeword $C=(L, R)$ and leak 
information from it. The leakage will be modeled by allowing the adversary to choose functions $\operatorname{Leak}_{i}^{\mathrm{L}}$ and $\operatorname{Leak}_{i}^{\mathrm{R}}$ and learn $\operatorname{Leak}_{i}^{\mathrm{L}}(L)$ and $\operatorname{Leak}_{i}^{\mathrm{R}}(R)$ (respectively). The entire process should happen in several rounds, and the adversary should be adaptive (i.e. his behavior in round $i$ should depend on what he learned in the previous rounds). The functions will be arbitrary, except that we will have a bound on the total number of bits leaked from $L$ and $R$, where the "number of leaked bits" is measured in terms of the total out size of the $\operatorname{Leak}_{i}^{\mathrm{L}}(L)$ and $\operatorname{Leak}_{i}^{\mathrm{L}}(R)$ functions. This is essentially the independent leakage model first considered in DP08 (inspired by the "only computation leaks" paradigm of Micali and Reyzin [MR04]) and then in a sequence of papers (see, e.g.: [DDV10, FKPR10, GR12, DF12, BDL14). It makes particular sense to use it in our context, as it is also motivated by the assumption that $L$ and $R$ are stored on two separate memory parts. Observe also that if we allowed joint leakage from $L$ and $R$ then we would need to have some additional restrictions on the leakage functions, as otherwise the adversary could choose a leakage function that first computes $M=\operatorname{Dec}(L, R)$ and then outputs the first bit $M[1]$ of $M$. This, in turn, would allow him to choose tampering functions that simply overwrite the original encoding with $\left(L^{\prime}, R^{\prime}\right)$ such that $\operatorname{Dec}\left(L^{\prime}, R^{\prime}\right)$ is equal to $(M[1], 0, \ldots, 0)$ (such $M^{\prime}$ is obviously "related" to $M$ and with overwhelming probability it is not equal to $M)$. For similar reasons it is obvious that we always need some sort of restriction on the leakage functions $\operatorname{Leak}_{i}^{\mathrm{L}}$ and $\operatorname{Leak}_{i}^{\mathrm{R}}$, since if the adversary learns the entire $L$ (say) then he can then easily choose a leakage function Leak ${ }_{i}^{\mathrm{R}}$ that first computes $\operatorname{Dec}(L, R)$ and then outputs $M[1]$.

It is also easy to see that without loss of generality we can restrict the adversary to choose deterministic leakage functions (since we can always convert a random function to a deterministic one by fixing its random input). Another natural observation is that it is enough to consider the case when all the leakage happens before the tampering functions are chosen. This is because the result of any leakage Leak $_{i}$ from a tampered codeword $f(C)$ can be computed by a function Leak $_{i}^{\prime}=$ Leak $_{i} \circ f$ that is applied directly to $C$. The formal definition of our model appears in Section 3.

\subsection{Our contribution}

As argued above, leakage-resilence is an important property for may applications of the nonmalleable codes. Unfortunately, this aspect of these codes has been ignored in many recent papers on this topic. In particular, the authors of [ADL14, CZ14 do not consider leakage at all. Leakageresilience was considered in [DKO13, but their construction works only for messages of length 1. To summarize: until now, no construction of leakage-resilient 2-split state non-malleable codes for messages longer than 1 was known. Providing such a construction is the main contribution of this work.

In fact, our contribution is much more general. We show a generic transformation that takes as input any non-malleable code (Enc, Dec) in the 2-split state model, and constructs out of it another non-malleable code $\left(\mathrm{Enc}^{\prime}\right.$, Dec' $)$ in the 2-split state model that additionally is secure against leaking a constant fraction of the bits from the codeword. The rate of $\left(\mathrm{Enc}^{\prime}, \mathrm{Dec}^{\prime}\right)$ is linear in the rate of (Enc, Dec). The only thing that we require is that (Enc, Dec) is symmetric, i.e., $\operatorname{Dec}(L, R)=$ $\operatorname{Dec}(R, L)$. Since the code of [ADL14 has this property, thus, combining this result with ours, we obtain a leakage-resilient 2 -split state non-malleable code.

Let us also note that the code from the subsequent work of [ADKO14] (built by applying a reduction from 10-split to 2-split state to the code of [CZ14]) is also symmetric, and therefore we can instantiate (Enc, Dec) with this construction, obtaining that $\left(E_{n c}^{\prime}\right.$, Dec $\left.^{\prime}\right)$ is a constant rate and can tolerate leakage of a linear size.

Our key technical argument is contained in Theorem 5.1 that can be of independent interest. 
Informally, in this theorem we consider a "parallel composition of the inner product encodings", i.e., we consider encoding of a pair of messages $x_{1}, x_{2} \in \mathbb{F}$ as random elements $L_{1}, R_{1}, L_{2}, R_{2} \in \mathbb{F}^{n}$ such that $\left\langle L_{1}, R_{1}\right\rangle=x_{1}$, and $\left\langle L_{2}, R_{2}\right\rangle=x_{2}$. We show that it is partly resilient to tampering in the following sense. If $\left(L_{1}, L_{2}\right)$ and $\left(R_{1}, R_{2}\right)$ are independently tampered to obtain $\left(L_{1}^{\prime}, L_{2}^{\prime}\right)$ and $\left(R_{1}^{\prime}, R_{2}^{\prime}\right)$, and then we decode to get $x_{1}^{\prime}=\left\langle L_{1}^{\prime}, R_{1}^{\prime}\right\rangle$, and $x_{2}^{\prime}=\left\langle L_{2}^{\prime}, R_{2}^{\prime}\right\rangle$, then $x_{1}^{\prime}, x_{2}^{\prime}$ can only have a limited dependence on $x_{1}, x_{2}$. The proof of this result is done using a careful combinatorial argument that among other techniques, makes extensive use of the two-source extractor property of the inner-product, and Vazirani's XOR Lemma.

\subsection{Comparison with Prior Work}

Techniques. The proof technique of our main technical result (Theorem 5.1) has some similarity with the results of prior work [DKO13, ADL14, CZ14]. We analyze the joint distribution

$$
\phi_{f, g}(L, R):=\left\langle L_{1}, R_{1}\right\rangle,\left\langle L_{2}, R_{2}\right\rangle,\left\langle f_{1}\left(L_{1}, L_{2}\right), g_{1}\left(R_{1}, R_{2}\right)\right\rangle,\left\{f_{2}\left(L_{1}, L_{2}\right), g_{2}\left(R_{1}, R_{2}\right)\right\}
$$

where $\left(L_{1}, L_{2}, R_{1}, R_{2}\right)$ is uniform over a set $\mathcal{L} \times \mathcal{R} \subset \mathbb{F}^{4 n}$, for some finite field $\mathbb{F}$, and $f_{1}, f_{2}: \mathcal{L} \mapsto \mathbb{F}^{n}$, $g_{1}, g_{2}: \mathcal{R} \mapsto \mathbb{F}^{n}$ are arbitrary functions.

The analysis of this result, and the main technical result of [DKO13, ADL14, CZ14] proceeds by partitioning the ambient space (which is $\mathcal{L} \times \mathcal{R}$ in our result) into appropriate subsets, and analyzing the required distribution over these subsets and then combining using (an equivalent of) Lemma 8.1. However, the partitioning procedure for our work, in our opinion, is relatively more delicate which helps us obtain the result with good parameters without relying on advanced techniques from additive combinatorics as was the case in [ADL14, CZ14].

Leakage-resilence. The main reason we are able to make our codes resilient to leakage is because, in Theorem 5.1. we analyze the distribution of $\phi_{f, g}(L, R)$, conditioned on $L, R$ being uniform in a subset of $\mathbb{F}^{2 n}$, and not the entire $\mathbb{F}^{2 n}$. This immediately leads to the question whether such an analysis over a subset of the ambient space would make the schemes of [ADL14, CZ14] also leakageresilient. However, the proof of [ADL14], in particular Lemma 8, which considers the case when $f$ is far from linear and $g$ is far from constant, will go through only if (in their notation) the subset has size bigger than $p^{2 n-t}$, where $t=\Theta\left(n^{1 / 6} / \log p\right)$. This will lead to the code being resilient to only $\Theta(t / n)=\Theta\left(n^{-5 / 6} / \log p\right)$ fraction of leakage, as compared to a constant fraction in our result. On the contrary, the proof of [CZ14] will work even if we restrict to a subset of the ambient space, but this will likely lead to a very small leakage rate. Also, their code is non-malleable in the 10-split-state model, and hence is less practical than the 2-split-state model that we consider, and for this reason we did not pursue this approach.

\subsection{Other related work}

The notion of non-malleability was introduced in cryptography by Dwork et al. DDN98. Formal treatment of the tampering attacks was initiated in [IPSW06, GLM ${ }^{+}$04]. The non-malleable codes were also studied by Cheraghchi and Guruswami, who in CG14b show improved constructions of the non-malleable codes secure against bit-wise tampering and show a connection between the non-malleable codes and the seedless non-malleable extractors (which is a new notion that they introduce). In CG14a the same authors study the problem of the capacity of non-malleable codes secure against different (non-split-state) families. Extensions of non-malleable codes to the case of continuous tampering were studied in FMNV14a]. Non malleable codes secure against tampering 
functions coming from restricted complexity classes were studied in [FMVW14], and secure against the linear tampering functions were considered in [CCP12].

Simultaneous leakage and tampering attacks were also considered in [LL10] (who consider a more restricted type of leakage, called the "probing attacks"), in [KKS11] who construct tamperand leakage-resilient encryption and signature schemes, and in DK12] who show a general way to transform any cryptographic functionality into one that is secure against tampering with individual bits, and leaking a logarithmic amount of information. Probabilistic tampering attacks on boolean circuits (where the adversary can tamper each wire with a certain probability) were also considered in FPV11.

\section{Preliminaries}

For a set $T$, let $U_{T}$ denote a uniform distribution over $T$, and, for an integer $\ell$, let $U_{\ell}$ denote uniform distribution over $\ell$ bit strings. The statistical distance between two random variables $A, B$ is defined by $\Delta(A, B)=\frac{1}{2} \sum_{v}|\operatorname{Pr}[A=v]-\operatorname{Pr}[B=v]|$. We use $A \approx_{\varepsilon} B$ as shorthand for $\Delta(A, B) \leq \varepsilon$.

Lemma 2.1. For any (randomized) function $\alpha$, if $\Delta(A, B) \leq \varepsilon$, then $\Delta(\alpha(A), \alpha(B)) \leq \varepsilon$.

The min-entropy of a random variable $W$ is $\mathbf{H}_{\infty}(W) \stackrel{\text { def }}{=}-\log \left(\max _{w} \operatorname{Pr}[W=w]\right)$, and the conditional min-entropy of $W$ given $Z$ is $\mathbf{H}_{\infty}(W \mid Z) \stackrel{\text { def }}{=}-\log \left(\mathbb{E}_{z \leftarrow Z} \max _{w} \operatorname{Pr}[W=w \mid Z=z]\right)$ !

Definition 2.2. We say that an efficient function Ext : $\{0,1\}^{n} \times\{0,1\}^{n} \rightarrow\{0,1\}^{m}$ is an $(n, k, m, \varepsilon)$-two-source extractor [CG88] if for all independent sources $X, Y \in\{0,1\}^{n}$ such that minentropy $\mathbf{H}_{\infty}(X)+\mathbf{H}_{\infty}(Y) \geq k$, we have $(Y, \operatorname{Ext}(X, Y)) \approx_{\varepsilon}\left(Y, U_{m}\right)$, and $(X, \operatorname{Ext}(X, Y)) \approx_{\varepsilon}\left(X, U_{m}\right)$.

For $n$ being an integer multiple of $m$, and interpreting elements of $\{0,1\}^{m}$ as elements from $\mathbb{F}_{2^{m}}$ and those in $\{0,1\}^{n}$ to be from $\left(\mathbb{F}_{2^{m}}\right)^{n / m}$, we have that the inner product function defined as $\left\langle\left(a_{1}, \ldots, a_{n / m}\right),\left(b_{1}, \ldots, b_{n / m}\right)\right\rangle:=a_{1} b_{1}+\cdots+a_{n / m} b_{n / m}$ is a good 2-source extractor (cf. eg. [CG88, Rao07]).

Lemma 2.3. For all positive integers $m, n$ such that $n$ is a multiple of $m$, and for all $\varepsilon>0$, there exists an efficient $\left(n, n+m+2 \log \left(\frac{1}{\varepsilon}\right), m, \varepsilon\right) 2$-source extractor.

We will need the following results. The proofs of these results can be found in Appendix $\mathrm{A}$ for completeness. The following is a simple result from [ADL14].

Lemma 2.4. Let $X_{1}, Y_{1} \in \mathcal{A}_{1}$, and $X_{2}, Y_{2} \in \mathcal{A}_{2}$ be random variables such that $\Delta\left(\left(X_{1}, X_{2}\right) ;\left(Y_{1}, Y_{2}\right)\right) \leq$ $\varepsilon$. Then, for any non-empty set $\mathcal{A}^{\prime} \subseteq \mathcal{A}_{1}$, we have

$$
\Delta\left(X_{2}\left|X_{1} \in \mathcal{A}^{\prime} ; Y_{2}\right| Y_{1} \in \mathcal{A}^{\prime}\right) \leq \frac{2 \varepsilon}{\operatorname{Pr}\left(X_{1} \in \mathcal{A}^{\prime}\right)} .
$$

A leakage oracle is a machine $\Omega$ that takes as input $(L, R) \in\{0,1\}^{n} \times\{0,1\}^{n}$ and then answers the leakage queries of a type $(\mathrm{L}, f)$ and $(\mathrm{R}, g)$, where $f, g:\{0,1\}^{n} \rightarrow\{0,1\}^{*}$. Each query $\left(\mathrm{L}, f_{i}\right)$ (resp.: $\left(\mathrm{R}, g_{i}\right)$ ) is answered with $f_{i}(L)$ (resp.: $g_{i}(R)$ ) or $\perp$. An interactive machine $\mathcal{A}$ that issues the leakage queries is called a leakage adversary. Let $\operatorname{Leak}_{\mathrm{L}}^{\mathcal{A}}(L)\left(\operatorname{resp}_{\mathrm{f}}\right.$ - $\left.\operatorname{Leak}_{\mathrm{R}}^{\mathcal{A}}(R)\right)$ denote the concatenation of all the non- $\perp$ answers to the $\left(\mathrm{L}, f_{i}\right)$ (resp.: $\left.\left(\mathrm{R}, g_{i}\right)\right)$ queries of $\mathcal{A}$. Moreover, let $\operatorname{Leak}^{\mathcal{A}}(L, R):=\left(\operatorname{Leak}_{\mathrm{L}}^{\mathcal{A}}(L), \operatorname{Leak}_{\mathrm{R}}^{\mathcal{A}}(R)\right)$. The oracle $\Omega$ is $m$-bounded if it gives the non- $\perp$ answers to the $\left(\mathrm{L}, f_{i}\right)$ queries as long as $\left|\operatorname{Leak}_{\mathrm{L}}^{\mathcal{A}}(L)\right| \leq m$ and the non- $\perp$ answers to $\left(\mathrm{R}, f_{i}\right)$ queries as long as $\left|\operatorname{Leak}_{\mathrm{R}}^{\mathcal{A}}(R)\right| \leq m$. The following result follows easily Lemma 4 of [DP07] and Lemma 2.2 of DORS08].

\footnotetext{
${ }^{1}$ Note that we use the variant of conditional entropy where the logarithm is taken after $\max _{w}$ is determined. This definition was introduced in DORS08] (it was called an average min-entropy there).
} 
Lemma 2.5. Let $\tilde{L}$ and $\tilde{R}$ be two independent random variables uniformly distributed respectively on sets $\mathcal{L} \subseteq\{0,1\}^{n}$ and $\mathcal{R} \subseteq\{0,1\}^{n}$ and let $\mathcal{A}$ be an arbitrary leakage adversary interacting with an $m$-bounded oracle $\Omega(\tilde{L}, \tilde{R})$. Then $\tilde{L}$ and $\tilde{R}$ are independent given $\operatorname{Leak}^{\mathcal{A}}(\tilde{L}, \tilde{R})$. Moreover, for every $\delta>0$ we have

$$
\operatorname{Pr}\left(\mathbf{H}_{\infty}\left(\tilde{L} \mid \operatorname{Leak}^{\mathcal{A}}(\tilde{L}, \tilde{R})=a\right) \leq \log |\mathcal{L}|-m-\log (1 / \delta)\right) \leq \delta
$$

(where $\left.a:=\operatorname{Leak}^{\mathcal{A}}(\tilde{L}, \tilde{R})\right)$, and

$$
\operatorname{Pr}\left(\mathbf{H}_{\infty}\left(\tilde{R} \mid \operatorname{Leak}^{\mathcal{A}}(\tilde{L}, \tilde{R})=b\right) \leq \log |\mathcal{L}|-m-\log (1 / \delta)\right) \leq \delta,
$$

(where $\left.b:=\operatorname{Leak}^{\mathcal{A}}(\tilde{L}, \tilde{R})\right)$.

We say that $\left(\right.$ Enc $_{\mathrm{LR}}: \mathcal{M} \rightarrow \mathcal{L} \times \mathcal{R}$, Dec $\mathrm{CR}_{\mathrm{LR}}: \mathcal{L} \times \mathcal{R} \rightarrow \mathcal{M}$ ) is an $\varepsilon$-leakage-resilient encoding in the split-state model [DDV10] if for every $M_{0}, M_{1} \in \mathcal{M}$ we have that

$$
\Delta\left(\operatorname{Leak}\left(\operatorname{Enc}_{\mathrm{LR}}\left(M_{0}\right)\right) ; \operatorname{Leak}\left(\operatorname{Enc}_{\mathrm{LR}}\left(M_{1}\right)\right) \leq \varepsilon .\right.
$$

Such encodings can be easily constructed from the 2-source extractors [DDV10. The following is a generalization of the Vazirani's XOR Lemma (it is proven in Appendix A).

Lemma 2.6. Let $X=\left(X_{1}, \ldots, X_{t}\right) \in \mathbb{F}^{t}$ be a random variable, where $\mathbb{F}$ is a finite field of order $q$. Assume that for all $a_{1}, \ldots, a_{t} \in \mathbb{F}^{t}$ not all zero, $\Delta\left(\sum_{i=1}^{t} a_{i} X_{i} ; U\right) \leq \varepsilon$, where $U$ is uniform in $\mathbb{F}$. Then $\Delta\left(X_{1}, \ldots, X_{t} ; U_{1}, \ldots, U_{t}\right) \leq \varepsilon q^{t}$, where $U_{1}, \ldots, U_{t}$ are independent and uniform in $\mathbb{F}^{t}$.

\section{The defintion of the Leakage Resilient Non-Malleable Codes}

In this section we present the defintion of the leakage resilient non-malleable codes in the splitstate model. We first recall the definition of non-malleable codes in the split-state model from DPW10, ADL14. As discussed already informally the introduction in this model we assume that the codeword is split into two parts which are tampered independently.

Definition 3.1. A coding scheme in the split-state model consists of two functions: a randomized encoding function Enc : $\mathcal{M} \mapsto \mathcal{L} \times \mathcal{R}$, and a deterministic decoding function $\operatorname{Dec}: \mathcal{L} \times \mathcal{R} \mapsto \mathcal{M} \cup\{\perp\}$ such that, for each $M \in \mathcal{M}, \operatorname{Pr}(\operatorname{Dec}(\operatorname{Enc}(M))=M)=1$ (over the randomness of the encoding algorithm). Suppose $\mathcal{L}=\mathcal{R}$ and denote $\mathcal{C}:=\mathcal{L}(=\mathcal{R})$. A coding scheme (Enc, Dec) is symmetric if for every $(L, R) \in \mathcal{C}$ we have that $\operatorname{Dec}(L, R)=\operatorname{Dec}(R, L)$.

Definition 3.2. Let $\left(\mathrm{Enc}: \mathcal{M} \rightarrow \mathcal{C}^{2}\right.$, Dec $\left.: \mathcal{C}^{2} \rightarrow \mathcal{M}\right)$ be a coding scheme in a split state model. For tampering functions $f, g: \mathcal{C} \rightarrow \mathcal{C}$, and $m \in \mathcal{M}$, define the tampering-experiment

$$
\operatorname{Tamper}_{m}:=\left\{\begin{array}{c}
(L, R) \leftarrow \operatorname{Enc}(m), \\
(\tilde{L}, \tilde{R}):=(f(L), g(R)) \\
\tilde{m}=\operatorname{Dec}(\tilde{L}, \tilde{R}) \\
\text { Output: } \tilde{m},
\end{array}\right\}
$$

which is a random variable over the randomness of the encoding function Enc. We say that a coding scheme (Enc, Dec) is $\varepsilon$-non-malleable w.r.t. $\mathcal{F}$ if for each $f \in \mathcal{F}$, there exists a distribution (corresponding to the simulator) $D$ over $\mathcal{M} \cup\{\perp$, same $\}$, such that, for all $m \in \mathcal{M}$, we have that the statistical distance between Tamper $_{m}$ and

$$
\operatorname{Sim}_{m}:=\left\{\begin{array}{c}
\tilde{m} \leftarrow D \\
\text { Output: } m \text { if } \tilde{m}=\text { same, and } \tilde{m} \text {, otherwise }
\end{array}\right\}
$$

is at most $\varepsilon$. Additionally, $D$ should be efficiently samplable given oracle access to $f$ and $g$. 
We now define the notion of non-malleability against the leakage adversaries (which was first formulated by [LL12]). As explained in the introduction it is enough to consider the scenario where the adversary first learns some bounded information about the codeword (via the leakage oracle), and then chooses the tampering functions. Formally we have the following.

Definition 3.3. Let Enc, Dec be a coding scheme from $\{0,1\}^{k}$ to $\{0,1\}^{n} \times\{0,1\}^{n}$, and let $\gamma \in[0,1)$ be a parameter. Let $\mathcal{A}$ be any adversary that has oracle access to a $\gamma n$-bounded leakage oracle $\Omega(L, R)$ (cf. 2), where $L, R \in\{0,1\}^{n}$, and outputs functions $(f, g)$ such that $f, g:\{0,1\}^{n} \rightarrow\{0,1\}^{n}$. Let $m \in\{0,1\}^{k}$ be a message. Consider the following tampering experiment.

$$
\operatorname{Tamper}_{m}^{\gamma}:=\left\{\begin{array}{c}
(L, R) \leftarrow \operatorname{Enc}(m), \\
(f, g)=\mathcal{A}(\Omega(L, R)), \\
(\tilde{L}, \tilde{R}):=(f(L), g(R)), \\
\tilde{m}=\operatorname{Dec}(\tilde{L}, \tilde{R}) \\
\text { Output: } \tilde{m},
\end{array}\right\}
$$

which is a random variable over the randomness of the encoding function Enc. We say that a coding scheme (Enc, Dec) is $\gamma$-leakage resilient $\varepsilon$-non-malleable code if for each $\mathcal{A}$, there exists a distribution $D$ over $\{0,1\}^{k} \cup\{\perp$, same $\}$, such that, for all $m \in\{0,1\}^{k}$, we have that the statistical distance between Tamper $_{m}^{\gamma}$ and

$$
\operatorname{Sim}_{m}:=\left\{\begin{array}{c}
\tilde{m} \leftarrow D \\
\text { Output: } m \text { if } \tilde{m}=\text { same, and } \tilde{m} \text {, otherwise. }
\end{array}\right\}
$$

is at most $\varepsilon$. Additionally, $D$ should be efficiently samplable given oracle access to $f, g$, Leak , Leak $_{2}$.

Of course, every $\varepsilon$-non-malleable code is also a 0-leakage resilient $\varepsilon$-non-malleable code. On the other hand, it is easy to find codes that are $\varepsilon$-non-malleable but are not $\xi$-leakage resilient $\varepsilon$-nonmalleable for an arbitrarily small $\xi$. For example, consider an $\varepsilon$-non-malleable code (Enc, Dec), and construct another code $\left(\mathrm{Enc}^{\prime}, \mathrm{Dec}^{\prime}\right)$ as follows. Let

$$
\operatorname{Enc}^{\prime}(M)=(\underbrace{(L, \cdots, L)}_{\lceil 1 / \xi\rceil \text { times }}, \underbrace{(R, \cdots, R)}_{\lceil 1 / \xi\rceil \text { times }}),
$$

where $(L, R) \leftarrow \operatorname{Enc}(M)$. The decoding function for $\left(L^{\prime}, R^{\prime}\right)=\left(\left(L_{1}, \ldots, L_{\lceil 1 / \xi\rceil}\right),\left(\left(R_{1}, \ldots, R_{\lceil 1 / \xi\rceil}\right)\right.\right.$ is defined as: $\operatorname{Dec}^{\prime}\left(L^{\prime}, R^{\prime}\right)=\perp$ if for some $i, j$ we have $L_{i} \neq L_{j}$ or $R_{i} \neq R_{j}$, and $\operatorname{Dec}^{\prime}\left(L^{\prime}, R^{\prime}\right)=$ $\operatorname{Dec}\left(L_{1}, R_{1}\right)$ otherwise. It is easy to show that $\left(\mathrm{Enc}^{\prime}, \mathrm{Dec}^{\prime}\right)$ is also $\varepsilon$-non-malleable. On the other hand, clearly, it is not $\xi$-leakage resilient $\varepsilon$-non-malleable, since the adversary can simply leak $L_{i}$ and $R_{i}$ (since $\left|L_{i}\right| /\left|L^{\prime}\right|=\left|R_{i}\right| /\left|R^{\prime}\right|=1 /(\lceil 1 / \xi\rceil) \leq \xi$ ) and hence he can compute $M$ before he chooses the tampering functions. Actually, it would even be enough for the adversary to leak $L_{i}$ from one part $(L$, say), since in this case he could make the tampering function $g$ fully dependent on $M$ (since $M=\operatorname{Dec}\left(L_{1}, R_{1}\right)$ ), and, e.g., tamper with $R$ only if $M=0$ (which obviously means that the code is malleable).

\section{Our construction}

This section contains the main construction of our paper. Let (Enc: $\mathcal{M} \rightarrow \mathcal{X} \times \mathcal{X}$, Dec : $\mathcal{X} \times \mathcal{X} \rightarrow$ $\mathcal{M})$ be a (not leakage resilient) symmetric $\varepsilon$-non-malleable code in the split state model. Let $\gamma \in[0,1 / 12)$ be a parameter. We are going to construct a $\gamma$-leakage resilient $3 \varepsilon$-non-malleable code $\left(E_{n c}^{\prime}, D_{e c}^{\prime}\right)$ in the split state mode (the code $\left(E_{n c}^{\prime}, D^{\prime} c^{\prime}\right)$ will also be symmetric). 
The first obvious idea for constructing such a code could be to define $\operatorname{Enc}^{\prime}(m)$ as follows: first compute $\left(x_{1}, x_{2}\right)=\operatorname{Enc}(m)$, and then "encode" both $x_{1}$ and $x_{2}$ using the leakage-resilient encoding (cf. Section 2). To be more concrete choose an encoding of [DDV10] that is based on the inner product function 2 . Let $\left(\ell_{1}, r_{1}\right)$ and $\left(\ell_{2}, r_{2}\right)$ be such leakage-resilient encodings of $x_{1}$ and $x_{2}$, respectively. It is clear that given $\left(\ell_{1}, r_{1}, \ell_{2}, r_{2}\right)$ one can easily compute $m$ as $\operatorname{Dec}\left(\left\langle\ell_{1}, r_{1}\right\rangle,\left\langle\ell_{2}, r_{2}\right\rangle\right)$.

Of course, what remains to be defined is how the variables $\ell_{1}, r_{1}, \ell_{2}$, and $r_{2}$ are represented in the final encoding, or, in other words: on which memory part one would store each of these variables. One option, of course, would be to move to the 4-split state model and say that the result of the encoding is $\left(\ell_{1}, r_{1}, \ell_{2}, r_{2}\right)$ (i.e. each of these variables can leak and be tampered independently). This approach can be proven secure, but it is clearly suboptimal since it increases to 4 the number of memory parts needed to implement the scheme $3^{3}$

If we restrict ourselves to the 2 -split state model then we could simply think of putting some of the $\ell_{1}, \ell_{2}, r_{1}, r_{2}$ variables on one part of the encoding and the remaining ones on the other part. It is easy to see that $\ell_{1}$ and $r_{1}$ cannot be put together on one memory part (a symmetric argument works for $\ell_{2}$ and $r_{2}$ ). This is because if the leakage function can be applied directly to $\ell_{1}$ and $r_{1}$ then the adversary choose a function that it simply internally decodes $x_{1}$ from $\left(\ell_{1}, r_{1}\right)$ and leaks directly from $x_{1}$. Hence, the code would need to be non-malleable even if the adversary can choose the tampering function $g$ (that is applied to $x_{2}$ ) after learning $x_{1}$, which is impossible (cf. the discussion at the end of Section 3).

Hence, the only option that has chances to work is to define the encoding of $m$ to be equal to $\left(\left(\ell_{1}, \ell_{2}\right),\left(r_{1}, r_{2}\right)\right)$. Observe that the adversary can now obviously "swap" $x_{1}$ and $x_{2}$ by changing the encoding to $\left(\left(\ell_{2}, \ell_{1}\right),\left(r_{2}, r_{1}\right)\right)$. This is ok for us, since we assumed that our encoding is symmetric. He can also "copy" elements, and produce an encoding $\left(\left(\ell_{1}, \ell_{1}\right),\left(r_{1}, r_{1}\right)\right)$ (or $\left.\left(\left(\ell_{2}, \ell_{2}\right),\left(r_{2}, r_{2}\right)\right)\right)$. In this case the decoded value $\left(x_{1}^{\prime}, x_{2}^{\prime}\right)$ will be equal to $\left(x_{1}, x_{1}\right)$ (resp.: $\left.\left(x_{2}, x_{2}\right)\right)$. It turns out that this is also ok, since every non-malleable code in the 2 -split state is essentially also a 2-out-of-2 secret sharing function (we prove this fact in Lemma 6.1), and thus $x_{1}$ and $x_{2}$ individually do not provide any significant information about the encoded message $m$ (which, of course, implies that $\operatorname{Dec} \operatorname{NM}_{\mathrm{NM}}\left(x_{1}, x_{1}\right)$ is unrelated to $m$ ). Of course these attacks can be combined with tampering attacks applied to each of $\ell_{1}, \ell_{2}, r_{1}$ and $r_{2}$ individually. For example the adversary can transform the encoding to $\left(\left(\ell_{1}, c \cdot \ell_{1}\right),\left(r_{1}, r_{1}\right)\right)$, which (from the linearity of the inner product) would decode to $\left(x_{1}, c \cdot x_{1}\right)$. This, however, is not a problem, since such individual tampering is obviously tolerated by every non-malleable code.

Unfortunately, it turns out the the adversary can launch some more sophisticated attacks. Observe that in our encoding the values $\left(\ell_{1}, \ell_{2}\right)$ and $\left(r_{1}, r_{2}\right)$ can be treated as vectors of length $2 n$. Suppose the adversary permutes both of them with the same random permutation $\sigma$, and let $\left(\ell_{1}^{\prime}, \ell_{2}^{\prime}\right)=\sigma\left(\ell_{1}, \ell_{2}\right)$ and $\left(r_{1}^{\prime}, r_{2}^{\prime}\right)=\sigma\left(r_{1}, r_{2}\right)$. Then the inner product of the $2 n$-long vectors remains unchanged (i.e. $\left.\left\langle\left(\ell_{1}^{\prime}, \ell_{2}^{\prime}\right),\left(r_{1}^{\prime}, r_{2}^{\prime}\right)\right\rangle=\left\langle\left(\ell_{1}, \ell_{2}\right),\left(r_{1}, r_{2}\right)\right\rangle\right)$, and therefore $\left\langle\ell_{1}, r_{1}\right\rangle+\left\langle\ell_{2}, r_{2}\right\rangle=$ $\left\langle\ell_{1}^{\prime}, r_{1}^{\prime}\right\rangle+\left\langle\ell_{2}^{\prime}, r_{2}^{\prime}\right\rangle$, which means that $x_{1}^{\prime}+x_{2}^{\prime}=x_{1}+x_{2}$ (where $x_{1}^{\prime}$ and $x_{2}^{\prime}$ are the results of decoding the manipulated encodings). Since $\left\langle\ell_{1}^{\prime}, r_{1}^{\prime}\right\rangle$ is uniformly random, thus one can think of this attack as $\left(x_{1}^{\prime}, x_{2}^{\prime}\right):=\left(x_{1}+Z, x_{2}-Z\right)$ for some random $Z$. Fortunately, the non-malleable codes are obliviously secure against attacks that add and subtract constants to the different parts of the encoding. Nevertheless, this example indicates that analyzing all possible strategies of the adversary may be non-trivial.

\footnotetext{
${ }^{2}$ In this encoding in order to encode a message $x$ one chooses random vectors $\ell$ and $r$ (in some $\mathbb{F}^{n}$ ) such that $\langle\ell, r\rangle=x$, and to decode the message one simply computes $\langle\ell, r\rangle$.

${ }^{3}$ One can be tempted to say that in this case we can apply the transformation from the subsequent paper [ADKO14] to reduce the number of parts from 4 to 2, but for this approach to work one would need to show that the construction of ADKO14 preserves the leakage-resilience which seems highly non-trivial.
} 
In Section 5 we characterize all such strategies by dividing them into classes. Very roughly speaking, it turns out that the attacks described above are examples of attacks from each of these classes. Namely: the adversary can either make $x_{1}^{\prime}$ depend only on $x_{1}$ and $x_{2}^{\prime}$ on $x_{2}$ (this case is denoted $\mathcal{D}_{\text {id }}$ ), or $x_{1}^{\prime}$ depend on $x_{2}$ and $x_{2}^{\prime}$ on $x_{1}$ (case $\mathcal{D}_{\text {swap }}$ ), or make both $x_{1}^{\prime}$ and $x_{2}^{\prime}$ depend only on $x_{1}$ (case $\mathcal{D}_{\text {forget }, 2}$ ) or only on $x_{2}$ (case $\mathcal{D}_{\text {forget, } 1}$ ). He can also make $x_{1}^{\prime}$ depend in an arbitrary way on $x_{1}, x_{2}$ and $x_{2}^{\prime}$ (case $\mathcal{D}_{\text {unif,2 }}$ ). This comes at a cost of making $x_{1}, x_{2}$ and $x_{2}^{\prime}$ uniform and independent (note that the " $\left(x_{1}^{\prime}, x_{2}^{\prime}\right):=\left(x_{1}+Z, x_{2}-Z\right)$ " attack falls into this category). Symmetrically, he can make $x_{2}^{\prime}$ depend in an arbitrary way on $x_{1}, x_{2}$ and $x_{1}^{\prime}$ (case $\mathcal{D}_{\text {unif, } 1}$ ).

What remains is to prove security of the non-malleable codes when the adversary can perform the attacks from classes $\mathcal{D}_{\text {id }}, \mathcal{D}_{\text {swap }}, \mathcal{D}_{\text {forget }, 1}, \mathcal{D}_{\text {forget }, 2}, \mathcal{D}_{\text {unif }, 1}$, and $\mathcal{D}_{\text {unif }, 2}$. This is done in Section 6 . In order to handle the cases $\mathcal{D}_{\text {unif, } 1}$, and $\mathcal{D}_{\text {unif }, 2}$ we need to modify our construction slightly. Namely, we make the leakage-resilient encoding "sparse" in the sense that a decoding of random codeword with overwhelming probability yields $\perp$ (this is slightly reminiscent of the construction of [DKO13] where a similar technique was used to construct the non-malleable codes for 1-bit messages). This is achieved by requiring that the decoded value has to be in some sparse subset of $\mathbb{F}$ of size $q^{\prime} \ll|\mathbb{F}|$. It will be convenient to define this set as the set of all $x$ 's such that $\psi(x) \leq q^{\prime}$, where $\psi: \mathbb{F} \rightarrow[q]$ is an arbitrary bijection. The technical details follow.

Our construction Let $n$ be an integer, and let $\mathbb{F}=\mathbb{F}_{q}$ be a finite field, and let $q^{\prime}<q$ be an integer. Let $\prec$ be a total order on $\mathbb{F}$, and let $\psi: \mathbb{F} \rightarrow[q]$ be a bijection such that $a \prec b$ if and only $\psi(a)<\psi(b)$, for all $a, b \in \mathbb{F}$. Define the "leakage-resilient" decoding function $\operatorname{Dec}_{\mathrm{LR}}: \mathbb{F}^{2 n} \times \mathbb{F}^{2 n} \rightarrow\left[q^{\prime}\right] \times\left[q^{\prime}\right] \cup\{\perp\}$ as follows:

$$
\operatorname{Dec}_{\mathrm{LR}}\left(\left(\ell_{1}, \ell_{2}\right),\left(r_{1}, r_{2}\right)\right)= \begin{cases}\perp & \text { if } \psi\left(\left\langle\ell_{1}, r_{1}\right\rangle\right)>q^{\prime}, \text { or } \psi\left(\left\langle\ell_{2}, r_{2}\right\rangle\right)>q^{\prime} \\ \left(\psi\left(\left\langle\ell_{1}, r_{1}\right\rangle\right), \psi\left(\left\langle\ell_{2}, r_{2}\right\rangle\right)\right) & \text { otherwise. }\end{cases}
$$

We then define $\operatorname{Enc}_{\mathrm{LR}}:\left[q^{\prime}\right] \times\left[q^{\prime}\right] \rightarrow \mathbb{F}^{2 n} \times \mathbb{F}^{2 n}$ as follows: for any $x \in\left[q^{\prime}\right] \times\left[q^{\prime}\right]$, $\operatorname{Enc}_{\mathrm{LR}}(x)$ is a random element $y$ in $\mathbb{F}^{2 n} \times \mathbb{F}^{2 n}$, such that $\operatorname{Dec}_{\mathrm{LR}}(y)=x$. The following is our main result. The proof is given in Section 6 .

Theorem 4.1. Let (Enc $\mathrm{EM}_{\mathrm{N}}$, Dec $\left.\mathrm{NM}\right)$ be an $\varepsilon$-non-malleable code from $\{0,1\}^{k}$ to $\left[q^{\prime}\right] \times\left[q^{\prime}\right]$ in the splitstate model (for any $\varepsilon \in(0,1 / 10)$ ) where Dec $\mathrm{NM}_{\mathrm{N}}$ is a symmetric function. Let $\left(\mathrm{Enc}_{\mathrm{LR}}\right.$, Dec $\mathrm{LR}$ ) be as above, with $q \geq \frac{q^{\prime}}{\varepsilon}$. Then for any $\gamma<\frac{1}{12}$ the encoding scheme $\left(\mathrm{Enc}_{\mathrm{LR}} \circ \mathrm{Enc}_{\mathrm{NM}}\right.$, $\left.\operatorname{Dec}_{\mathrm{NM}} \circ \operatorname{Dec}_{\mathrm{LR}}\right)$ is an efficient $3 \varepsilon$-non-malleable $\gamma$-leakage resilient code in the split-state model from $\{0,1\}^{k}$ to $\mathbb{F}_{q}^{n} \times \mathbb{F}_{q}^{n}$, where $n=O\left(\frac{1}{1 / 12-\gamma}\right)$.

Thus, using the result of ADL14, Agg14, we get the following result.

Corollary 4.2. For any $\gamma<\frac{1}{12}$ and any $\varepsilon \in(0,3 / 10)$, there exists an efficient $\gamma$-leakage-resilient $\varepsilon$-non-malleable code in the split-state model from $k$-bit messages to $\Theta\left(\frac{(k+\log (1 / \varepsilon))^{7}}{1 / 12-\gamma}\right)$-bit codewords.

Also, we get the following stronger result by combining Theorem 4.1 with an upcoming result [ADKO14, which gives a constant-rate non-malleable code in the split-state model.

Corollary 4.3. For any $\gamma<\frac{1}{12}$ and any $\varepsilon \in(0,3 / 10)$, there exists an efficient $\gamma$-leakage-resilient $\varepsilon$-non-malleable code in the split-state model from $k$-bit messages to $\Theta\left(\frac{k+\log (1 / \varepsilon)}{1 / 12-\gamma}\right)$-bit codewords. 


\section{$5 \quad$ The joint distribution of $\phi_{f, g}(L, R)$}

Let $\mathbb{F}=\mathbb{F}_{q}$ be a finite field. Let $\left(L_{1}, L_{2}\right),\left(R_{1}, R_{2}\right)$ be independent and distributed uniformly over $\mathcal{L}, \mathcal{R} \subseteq \mathbb{F}^{2 n}$, respectively. Let $f_{1}, g_{1}, f_{2}, g_{2}: \mathbb{F}^{n} \times \mathbb{F}^{n} \rightarrow \mathbb{F}^{n}$ be a pair of functions. We consider the following family of distributions

$$
\phi_{f, g}(L, R):=\left(\left\langle L_{1}, R_{1}\right\rangle,\left\langle L_{2}, R_{2}\right\rangle,\left\langle f_{1}\left(L_{1}, L_{2}\right), g_{1}\left(R_{1}, R_{2}\right)\right\rangle,\left\langle f_{2}\left(L_{1}, L_{2}\right), g_{2}\left(R_{1}, R_{2}\right)\right)\right\rangle \in \mathbb{F}^{4}
$$

In this section, we analyze the possible joint distribution of $\phi_{f, g}(L, R)$ over $\mathbb{F}^{4}$ for arbitrary functions $f_{1}, g_{1}, f_{2}, g_{2}$. First, define the following set of distributions (which were already informally discussed in Section 4).

- $\mathcal{D}_{\text {id }}:=\left\{\left(U_{1}, U_{2}, h_{1}\left(U_{1}, Z\right), h_{2}\left(U_{2}, Z\right)\right)\right\}$, where $h_{1}, h_{2}$ are functions from $\mathbb{F} \times \mathcal{Z}$ to $\mathbb{F}, U_{1}, U_{2}$ are independent and uniform in $\mathbb{F}$, and $Z \in \mathcal{Z}$ is some random variable independent of $U_{1}, U_{2}$.

- $\mathcal{D}_{\text {swap }}:=\left\{\left(U_{1}, U_{2}, h_{1}\left(U_{2}, Z\right), h_{2}\left(U_{1}, Z\right)\right)\right\}$, where $h_{1}, h_{2}$ are functions from $\mathbb{F} \times \mathcal{Z}$ to $\mathbb{F}, U_{1}, U_{2}$ are independent and uniform in $\mathbb{F}$, and $Z \in \mathcal{Z}$ is some random variable independent of $U_{1}, U_{2}$.

- $\mathcal{D}_{\text {unif, } 1}:=\left\{\left(U_{1}, U_{2}, U_{3}, W\right)\right\}$, where $U_{1}, U_{2}, U_{3}$ are independent and uniform in $\mathbb{F}$, and $W$ is a random variable over $\mathbb{F}$ arbitrarily correlated to $U_{1}, U_{2}, U_{3}$.

- $\mathcal{D}_{\text {unif }, 2}:=\left\{\left(U_{1}, U_{2}, W, U_{3}\right)\right\}$, where $U_{1}, U_{2}, U_{3}$ are independent and uniform in $\mathbb{F}$, and $W$ is a random variable over $\mathbb{F}$ arbitrarily correlated to $U_{1}, U_{2}, U_{3}$.

- $\mathcal{D}_{\text {forget }, 1}:=\left\{\left(U_{1}, U_{2}, h_{1}\left(U_{2}, Z\right), h_{2}\left(U_{2}, Z\right)\right)\right\}$, where $h_{1}, h_{2}$ are functions from $\mathbb{F} \times \mathcal{Z}$ to $\mathbb{F}, U_{1}, U_{2}$ are independent and uniform in $\mathbb{F}$, and $Z \in \mathcal{Z}$ is some random variable independent of $U_{1}, U_{2}$.

- $\mathcal{D}_{\text {forget }, 2}:=\left\{\left(U_{1}, U_{2}, h_{1}\left(U_{1}, Z\right), h_{2}\left(U_{1}, Z\right)\right)\right\}$, where $h_{1}, h_{2}$ are functions from $\mathbb{F} \times \mathcal{Z}$ to $\mathbb{F}, U_{1}, U_{2}$ are independent and uniform in $\mathbb{F}$, and $Z \in \mathcal{Z}$ is some random variable independent of $U_{1}, U_{2}$.

Define $\mathcal{D}$ to be the family of convex combinations of

$$
\mathcal{D}_{\text {id }} \cup \mathcal{D}_{\text {swap }} \cup \mathcal{D}_{\text {unif }, 1} \cup \mathcal{D}_{\text {unif }, 2} \cup \mathcal{D}_{\text {forget }, 1} \cup \mathcal{D}_{\text {forget }, 2} .
$$

We show that for any $f, g$, the value of $\phi_{f, g}(L, R)$ is statistically close to some distribution in $\mathcal{D}$ if $\mathcal{L}, \mathcal{R}$ have size at least $q^{2 n(1-\gamma)}$.

Theorem 5.1. Let $\mathbb{F}=\mathbb{F}_{q}$ be a finite field with $q \geq 4, n \geq 48$ be an integer, and $\gamma \in[0,1 / 12)$. Let $L=\left(L_{1}, L_{2}\right)$, and $R=\left(R_{1}, R_{2}\right)$ be distributed uniformly at random in sets $\mathcal{L}, \mathcal{R}$ of size at least $q^{2 n(1-\gamma)}$. For any $f_{1}, f_{2}, g_{1}, g_{2}: \mathbb{F}^{2 n} \rightarrow \mathbb{F}^{n}$, there exists a distribution $D \in \mathcal{D}$ such that

$$
\Delta\left(\phi_{f, g}(L, R) ; D\right) \leq 7^{2} \cdot 2^{-s} .
$$

for any $s \leq\left(\left(\frac{1}{12}-\gamma\right) n-\frac{5}{4}\right) \log q-\frac{5}{6}$.

We give a proof of this theorem in Section 8 .

\section{Concluding Theorem 4.1 from Theorem 5.1}

Before we present our proof, we state a result showing that non-malleable codes in 2 -split state model also have the secret sharing property, i.e., that given any one part of the encoding of $m$, it is impossible to guess the message $m$. The proof of this can be found in Appendix B. 
Lemma 6.1. Let $\operatorname{Dec}: \mathcal{X} \times \mathcal{X} \rightarrow \mathcal{M}$, and Enc $: \mathcal{M} \rightarrow \mathcal{X} \times \mathcal{X}$ be $\varepsilon$-non-malleable code in 2-split state model for some $\varepsilon<\frac{1}{2}$. For any pair of messages $m_{0}, m_{1} \in \mathcal{M}$, let $\left(X_{1}^{0}, X_{2}^{0}\right) \leftarrow \operatorname{Enc}\left(m_{0}\right)$, and let $\left(X_{1}^{1}, X_{2}^{1}\right) \leftarrow \operatorname{Enc}\left(m_{1}\right)$. Then $\Delta\left(X_{0} ; X_{1}\right) \leq 2 \varepsilon$.

Proof of Theorem 4.1. Let $n=\left\lceil\frac{6}{1 / 12-\gamma}\right\rceil$. Fix the message $m \in \mathcal{M}$, and let $\operatorname{Enc}_{\mathrm{NM}}(m)=\left(X_{1}, X_{2}\right)$, and $\operatorname{Enc}_{\mathrm{LR}}\left(X_{1}, X_{2}\right)=(L, R)$. Furthermore, fix manipulation functions $f, g: \mathbb{F}^{n} \times \mathbb{F}^{n} \rightarrow \mathbb{F}^{n} \times \mathbb{F}^{n}$ and the parameter $\gamma \in\left[0, \frac{1}{12}\right)$. We need to analyze the distribution Tamper ${ }_{m}^{\gamma}$ as in Definition 3.3. Before this, consider the following. Choose $\gamma^{\prime}=\frac{1 / 12+\gamma}{2}$, so that $\left(1 / 12-\gamma^{\prime}\right) n=\left(\gamma^{\prime}-\gamma\right) n \geq 3$. Let $\tilde{L}, \tilde{R}$ be uniform in $\mathbb{F}^{n}$. From Lemma 2.5 we know that the min-entropies of $\tilde{L}$ and $\tilde{R}$ conditioned on the knowledge of $\operatorname{Leak}^{\mathcal{A}}(\tilde{L}, \tilde{R})$ are at least $2 n \log q\left(1-\gamma^{\prime}\right)$ with probability at least $1-2 \cdot q^{2 n\left(\gamma-\gamma^{\prime}\right)}$. So, at the cost of at most $2 \cdot q^{2 n\left(\gamma-\gamma^{\prime}\right)}$ in the adversary's success probability, we can restrict ourselves to the case where $\tilde{L}$ and $\tilde{R}$ are distributed over uniformly over a set of size at least $q^{2 n\left(1-\gamma^{\prime}\right)}$. Consider the joint distribution

$$
\left\langle\tilde{L}_{1}, \tilde{R}_{1}\right\rangle,\left\langle\tilde{L}_{2}, \tilde{R}_{2}\right\rangle,\left\langle f_{1}\left(\tilde{L}_{1}, \tilde{L}_{2}\right), g_{1}\left(\tilde{R}_{1}, \tilde{R}_{2}\right)\right\rangle,\left\langle f_{2}\left(\tilde{L}_{1}, \tilde{L}_{2}\right), g_{2}\left(\tilde{R}_{1}, \tilde{R}_{2}\right)\right\rangle
$$

conditioned on the knowledge of $\operatorname{Leak}^{\mathcal{A}}(\tilde{L}, \tilde{R})$. By Theorem 5.1 , this has statistical distance at most

$$
49 \cdot 2^{5 / 6} \cdot q^{5 / 4-\left(1 / 12-\gamma^{\prime}\right) n}+2 \cdot q^{2 n\left(\gamma-\gamma^{\prime}\right)} \leq 100 q^{-7 / 4} \leq 100\left(\frac{\varepsilon^{2}}{2^{k}}\right)^{7 / 4} \leq \frac{\varepsilon}{2^{k+1}}
$$

from some distribution $D$ in $\mathcal{D}$. Here we used that $\varepsilon<1 / 10$, and $q^{\prime} \geq \frac{2^{k}}{\varepsilon}$. This holds for any non-malleable code in the 2 -split-state model. Note that for any known non-malleable code in this model (in particular, those used in Corollary 4.2 , and Corollary 4.3), we have that $q^{\prime} \gg \frac{2^{k}}{\varepsilon}$. Since $D$ is a convex combination (depending on $f, g$, and $\mathcal{A}$ ) of distributions in $\mathcal{D}_{\text {forget }, 1}, \mathcal{D}_{\text {forget }, 2}, \mathcal{D}_{\text {unif }, 1}$, $\mathcal{D}_{\text {unif }, 2}, \mathcal{D}_{\text {id }}$, and $\mathcal{D}_{\text {swap }}$, without loss of generality we will analyze the distribution Tamper ${ }_{m}^{\gamma}$ under the assumption that $D$ belongs to one of these sets. Hence, we consider the following cases.

$D \in \mathcal{D}_{\text {forget }, 1}:$ In this case, $D$ is of the form $U_{1}, U_{2}, h_{1}\left(U_{2}, Z\right), h_{2}\left(U_{2}, Z\right)$, where $U_{1}, U_{2}$ are independent and uniform in $\mathbb{F}$, and $Z$ is independent of $U_{1}, U_{2}$. Of course, in our case $L_{1}, R_{1}, L_{2}$ and $R_{2}$ are not entirely uniform and independent, as they are a random encoding of a fixed message $m$. To take it into account we use Lemma 2.4. that states that in this case the statistical distance gets mutiplied by 2 divided by the probability that a random message $M$ is equal to $m$ (which is equal to $2^{-k}$ ). Hence, we get that $\operatorname{Tamper}_{m}^{\gamma}$ has statistical distance at most

$$
2^{k+1} \cdot \frac{\varepsilon}{2^{k+1}}=\varepsilon
$$

from

$$
V_{1}=\operatorname{Dec}_{\mathrm{NM}}\left(\psi\left(h_{1}\left(\psi^{-1}\left(X_{2}\right), Z\right)\right), \psi\left(h_{2}\left(\psi^{-1}\left(X_{2}\right), Z\right)\right)\right),
$$

where $\operatorname{Enc}_{\mathrm{NM}}(m)=\left(X_{1}, X_{2}\right)$, and $Z$ is independent of $X_{1}, X_{2}$. Since $V_{1}$ is independent of $X_{1}$, using Lemma 6.1 we get the desired result (as $X_{2}$ cannot carry enough information to make $V_{1}$ dependent on $m$.

$D \in \mathcal{D}_{\text {forget }, 2}$ : This case is similar to the previous one.

$D \in \mathcal{D}_{\text {unif,1 }}$ : In this case, $D$ is of the form $U_{1}, U_{2}, U_{3}, W$, where $U_{1}, U_{2}, U_{3}$ are independent and uniform in $\mathbb{F}$, and $W \in \mathbb{F}$ is arbitrarily correlated to $U_{1}, U_{2}, U_{3}$. Again, by Lemma 2.4, this implies that Tamper $_{m}^{\gamma}$ has statistical distance at most

$$
2^{k+1} \cdot \frac{\varepsilon}{2^{k+1}}=\varepsilon
$$


from

$$
V_{2}=\operatorname{Dec}_{\mathrm{NM}}\left(\psi\left(U_{3}\right), W^{\prime}\right),
$$

where $\operatorname{Enc}_{\mathrm{NM}}(m)=\left(X_{1}, X_{2}\right), U_{3}$ is uniform and independent in $\mathbb{F}, W^{\prime}$ is arbitrarily correlated to $X_{1}, X_{2}, U_{3}$. Note that $\psi\left(U_{3}\right)=\perp$ with probability $1-q^{\prime} / q$. Thus, Tamper ${ }_{m}^{\gamma}$ has statistical distance at most $\varepsilon+q^{\prime} / q \leq 2 \varepsilon$ from $\perp$.

$D \in \mathcal{D}_{\text {unif }, 2}$ : This case is similar to the previous one.

$D \in \mathcal{D}_{\text {id }}$ : In this case, $D$ is of the form $U_{1}, U_{2}, h_{1}\left(U_{1}, Z\right), h_{2}\left(U_{2}, Z\right)$, where $U_{1}, U_{2}$ are independent and uniform in $\mathbb{F}$, and $Z$ is independent of $U_{1}, U_{2}$. By Lemma 2.4, this implies that Tamper ${ }_{m}^{\gamma}$ has statistical distance at most

$$
2^{k+1} \cdot \frac{\varepsilon}{2^{k+1}}=\varepsilon
$$

from

$$
V_{3}=\operatorname{Dec}_{\mathrm{NM}}\left(\psi\left(h_{1}\left(\psi^{-1}\left(X_{1}\right), Z\right)\right), \psi\left(h_{2}\left(\psi^{-1}\left(X_{2}\right), Z\right)\right)\right),
$$

where $\operatorname{Enc}_{\mathrm{NM}}(m)=\left(X_{1}, X_{2}\right)$, and $Z$ is independent of $X_{1}, X_{2}$. It is easy to see that $V_{3}$ is $\varepsilon$-close to $\operatorname{Sim}_{m}$ by the $\varepsilon$-non-malleability of (Enc $\mathrm{NM}_{\mathrm{NM}}$, $\left.\mathrm{Dec}_{\mathrm{NM}}\right)$.

$D \in \mathcal{D}_{\text {swap }}$ : Using the fact that the decoding function is symmetric, this case is similar to the previous one.

\section{Existential Result using [CG14b]}

Cheraghchi and Guruswami [CG14b] introduced the notion of seedless non-malleable extractors as a step towards constructing non-malleable codes defined as follows.

Definition 7.1. A function NMExt : $\{0,1\}^{n} \times\{0,1\}^{n} \rightarrow\{0,1\}^{k}$ is a two-source non-malleable $(m, \varepsilon)$-extractor if, for every pair of independent random variables $X, Y$ over $\{0,1\}^{n}$ such that $\mathbf{H}_{\infty}(X) \geq m$, and for any functions $f, g:\{0,1\}^{n} \rightarrow\{0,1\}^{n}$, there exists a distribution $D$ over $\{0,1\}^{k} \cup\{$ same $\}$, such that

$$
\Delta\left(\operatorname{NMExt}(X, Y), \operatorname{NMExt}\left(f(X), g(Y) ; U_{k}, \operatorname{copy}\left(D, U_{k}\right)\right) \leq \varepsilon,\right.
$$

where $U_{k}$ is uniformly random in $\{0,1\}^{k}$, and copy $\left(D, U_{k}\right)=U_{k}$ if $D=$ same, and $D$, otherwise.

It was shown in CG14b that assuming the existence of non-malleable extractors with $m=n$ immediately gives non-malleable codes with good rate. We observe that their proof easily extends to show that non-malleable extractors with small $m$ implies non-malleable codes with good rate that also tolerate large amount of leakage.

Theorem 7.2. Let NMExt : $\{0,1\}^{n} \times\{0,1\}^{n} \rightarrow\{0,1\}^{k}$ be a two-source non-malleable $(m, \varepsilon)$ extractor. Define a coding scheme (Enc, Dec) with message length $k$ and block length $2 n$ as follows. The decoder $\operatorname{Dec}$ is defined as $\operatorname{Dec}(x, y):=\operatorname{NMExt}(x, y)$.

The encoder, given a message s, outputs a uniformly random element $(X, Y)$ in $\{0,1\}^{n} \times\{0,1\}^{n}$ such that $\operatorname{Dec}(X, Y)=s$. Then the pair (Enc, Dec) is $\left(\varepsilon \cdot\left(2^{k}+1\right)\right.$-non-malleable, $\left.1-\frac{m}{n}\right)$-leakageresilient code against split-state tampering.

We now mention the result from [CG14b] showing the existence of non-malleable codes. 
Theorem 7.3. Let NMExt : $\{0,1\}^{n} \times\{0,1\}^{n} \rightarrow\{0,1\}^{k}$ be a random function. For any $\varepsilon, \delta>0$, and $m \leq n$, with probability at least $1-\delta$, the function NMExt is a two-source non-malleable extractor provided that

$$
m \geq \max \left(k+\frac{3}{2} \cdot \log 1 / \varepsilon+\frac{1}{2} \log \log (1 / \delta), \log n+\log \log (1 / \delta)+O(1)\right) .
$$

Combining Theorem 7.2 and Theorem 7.3 gives us the following corollary.

Corollary 7.4. Let $n=\operatorname{poly}(k)$, and let NMExt : $\{0,1\}^{n} \times\{0,1\}^{n} \rightarrow\{0,1\}^{k}$ be a random function. With probability at least $1-\frac{1}{2^{2^{k}}}$, the scheme $\left(\mathrm{NMExt}^{-1}, \mathrm{NMExt}\right)$ is $2^{-k}$-non-malleable, $1-\frac{5 k}{n}$-leakageresilient.

This implies that with probability very close to 1 , a random function is an excellent leakageresilient non-malleable code, and we can arbitrarily increase the amount of leakage (upto the total length of each part) at the cost of increasing the length of the codeword.

\section{Proof of Theorem 5.1}

\subsection{The general strategy}

Our strategy is to divide $\mathcal{L} \times \mathcal{R}$ into several disjoint parts, and prove that $\phi_{f, g}(L, R)$ is close to some distribution in $\mathcal{D}$ for each of these parts separately. Let $L=\left(L_{1}, L_{2}\right)$, and $R=\left(R_{1}, R_{2}\right)$. Also, let $|\mathbb{F}|=q$, and $\tau=1-\gamma$. The following simple lemma shows that it suffices to bound the statistical distance between $\phi_{f, g}(L, R)$ and some distribution in $\mathcal{D}$ for $(L, R)$ restricted to partitions of $\mathcal{L} \times \mathcal{R}$. This was shown in ADL14.

Lemma 8.1. Let $\mathcal{S} \subseteq \mathcal{L} \times \mathcal{R}$. Let $\mathcal{S}_{1}, \ldots, \mathcal{S}_{k}$ be a partition of $\mathcal{S}$. Also, let $D_{1}, \ldots, D_{k}$ be some distribution in $\mathcal{D}$. Assume that for all $1 \leq i \leq k$,

$$
\Delta\left(\left.\phi_{f, g}(L, R)\right|_{(L, R) \in \mathcal{S}_{i}} ; D_{i}\right) \leq \varepsilon_{i} .
$$

Then there exists a distribution $D \in \mathcal{D}$ such that

$$
\Delta\left(\left.\phi_{f, g}(L, R)\right|_{(L, R) \in \mathcal{S}} ; D\right) \leq \sum \varepsilon_{i} \frac{\left|\mathcal{S}_{i}\right|}{|\mathcal{S}|} .
$$

We construct a partition of $\mathcal{L} \times \mathcal{R}$ in Section 8.2. Then in Sections 8.3.1 8.3.4 we analyze the behavior of $\phi_{f, g}(L, R)$ on the constructed parts. Finally, in Section 8.4 we show how to combine the facts proven in previous sections in order to obtain the statement of the theorem.

\subsection{Partitioning the set $\mathcal{L} \times \mathcal{R}$.}

We next define a partitioning of $\mathcal{L} \times \mathcal{R}$ based on $f$ and $g$ to which we will apply Lemma 8.1. This is done independently for $\mathcal{L}$ and $\mathcal{R}$ and hence we will focus only on $\mathcal{L}$ (the partitioning of $\mathcal{R}$ is done analogously). On a high level, our partitioning is constructed as follows: first we partition $\mathcal{L}$ into sets $\mathcal{L}_{\text {ffb }, 1}, \mathcal{L}_{\text {ffb }, 2}$, and $\mathcal{L}_{1}$. Then we partition $\mathcal{L}_{1}$ into $\mathcal{L}_{\text {mix }, 1}, \mathcal{L}_{\text {mix }, 2}$, and $\mathcal{L}_{2}$. Finally, we partition $\mathcal{L}_{2}$ into $\mathcal{L}_{\text {id }}, \mathcal{L}_{\text {swap }}$, and $\mathcal{L}_{\text {rem }}$ (the meaning of the acronyms in the subscripts should become clear when the sets are defined). Altogether, we partition $\mathcal{L}$ into 7 sets $\mathcal{L}_{\text {ffb }, 1}, \mathcal{L}_{\text {ffb }, 2}, \mathcal{L}_{\text {mix }, 1}, \mathcal{L}_{\text {mix }, 2}, \mathcal{L}_{\text {id }}, \mathcal{L}_{\text {swap }}$ and $\mathcal{L}_{\text {rem }}$.

Let $\beta_{1}=\frac{1}{3} n \log q-4\left(\tau-\frac{11}{12}\right) n \log q+4 \log q+4 s+4$, and let $\beta_{2}=\frac{1}{3} n \log q-4\left(\tau-\frac{11}{12}\right) n \log q+$ $6 \log q+3 s+2$. We first partition $\mathcal{L}$ into $\mathcal{L}_{\text {ffb }, 1}, \mathcal{L}_{\text {ffb }, 2}$, and $\mathcal{L}_{1}$. Recall that the elements of $\mathcal{L}$ 
are pairs $\left(\ell_{1}, \ell_{2}\right) \in \mathbb{F}^{n} \times \mathbb{F}^{n}$. Intutively $\mathcal{L}_{\text {ffb }, i}$ (for $\left.i \in\{1,2\}\right)$ will consist of the elements of $\mathcal{L}$ on which the function $f$ is "far from a bijection", by which we mean that it "glues" at least $2^{\beta_{1} / 2}$ elements on the $i$ th component (cf. Steps 2 and 3 below). The set $\mathcal{L}_{1}$ will consists of the remaining elements (i.e. those that are "close to the bijection"). Since we want $\mathcal{L}_{\text {ffb }, 1}, \mathcal{L}_{\text {ffb }, 2}$, and $\mathcal{L}_{1}$ to be a partition, thus $\mathcal{L}_{\mathrm{ffb}, 1}$ and $\mathcal{L}_{\mathrm{ffb}, 2}$ have to be disjoint. Hence we first construct $\mathcal{L}_{\mathrm{ffb}, 1}$, and then, using the same method we construct $\mathcal{L}_{\mathrm{ffb}, 2}$, but in this construction we consider only $\ell$ 's that belong to $\mathcal{L}^{*}:=\mathcal{L} \backslash \mathcal{L}_{\text {ffb }, 1}$. The set $\mathcal{L}_{1}$ consits of the elements of $\mathcal{L}$ that were not included in $\mathcal{L}_{\text {ffb }, 1}$ or $\mathcal{L}_{\text {ffb }, 2}$. To avoid repetition, we present the procedures for constructing $\mathcal{L}_{\mathrm{ffb}, 1}$ and $\mathcal{L}_{\mathrm{ffb}, 2}$ as one algorithm, whose behaviour depends on $i$. This algorithm, presented below, is executed first for $i=1$ and then for $i=2$.

1. Initialize $\mathcal{L}_{\mathrm{ffb}, i}$ to be empty, and let $\mathcal{L}^{*}:=\mathcal{L}$ if $i=1$, and $\mathcal{L}^{*}:=\mathcal{L} \backslash \mathcal{L}_{\mathrm{ffb}, 1}$, otherwise.

2. Let $\mathcal{W}$ be a largest subset of $\mathcal{L}^{*}$ such that for any two $\ell, \ell^{\prime} \in \mathcal{W}$ it holds that $\ell_{i} \neq \ell_{i}^{\prime}$, and $f(\ell)=f\left(\ell^{\prime}\right)$.

3. If $|\mathcal{W}| \geq 2^{\beta_{1} / 2}$, then set $\mathcal{L}^{*}=\mathcal{L}^{*} \backslash \mathcal{W}$, set $\mathcal{L}_{\mathrm{ffb}, i}=\mathcal{L}_{\mathrm{ffb}, i} \cup \mathcal{W}$, and go to Step 2 .

4. Return $\mathcal{L}_{\text {ffb }, i}$.

The set $\mathcal{L}_{1}$ is defined to be

$$
\mathcal{L}_{1}=\mathcal{L} \backslash\left(\mathcal{L}_{\text {ffb }, 1} \cup \mathcal{L}_{\text {ffb }, 2}\right) .
$$

The justification for this choice is that for $\tilde{L}$ chosen uniformly at random from $\mathcal{L}_{\text {ffb }, i}$, we have

$$
\mathbf{H}_{\infty}\left(\tilde{L}_{i} \mid f(\tilde{L})=y\right) \geq \frac{\beta_{1}}{2}
$$

where $y:=f(\tilde{L})$ (this is because the number of pre-images of $y$ under $f$, projected on the $i$ th component, is at least $2^{\beta_{1} / 2}$ ). Also, we have that for any $y \in \mathbb{F}^{n}$, the total number of elements $\ell \in \mathcal{L}_{1}$ such that $f(\ell)=y$ is at most $\left(2^{\frac{\beta_{1}}{2}}\right)^{2}=2^{\beta_{1}}$.

Recall that every $f: \mathbb{F}^{n} \times \mathbb{F}^{n} \rightarrow \mathbb{F}^{n} \times \mathbb{F}^{n}$ can be represened as a pair of functions $f_{1}, f_{2}$ : $\mathbb{F}^{n} \times \mathbb{F}^{n} \rightarrow \mathbb{F}^{n}$ defined as $\left(f_{1}(\ell), f_{2}(\ell)\right):=f(\ell)$. We further partition the set $\mathcal{L}_{1}$ depending on how $f_{1}(\ell), f_{2}(\ell)$ depend on $\ell_{1}, \ell_{2}$ for $\ell \in \mathcal{L}_{1}$. We will now define partitioning of $\mathcal{L}_{1}$. Before we do it, let us state the following auxiliary definition (note that it is defined for $\mathcal{L}$, not for $\mathcal{L}_{1}$ ).

Definition 8.2. Define $T^{i \rightarrow j} \subset \mathcal{L}$ for $i, j \in\{1,2\}$, as the set of all elements $\ell \in \mathcal{L}$ such that

$$
\mid\left\{\ell^{*} \in \mathcal{L} \mid \ell_{i}=\ell_{i}^{*} \text { and } f_{j}(\ell)=f_{j}\left(\ell^{*}\right)\right\} \mid \geq \frac{q^{n}}{2^{\beta_{2}}} .
$$

Let us now prove the following simple result justifying the definition of $T^{i \rightarrow j}$. Intuitively, this result shows that for every $\ell \in T^{i \rightarrow j}$, the value of $f_{j}(\ell)$ can be computed given $\ell_{i}$ and a little more information.

Lemma 8.3. Let $\ell \in T^{i \rightarrow j}$ for some $i, j \in[t]$. Then there exists some functions $a_{i, j}: T^{i \rightarrow j} \mapsto$ $\{0,1\}^{\beta_{2}}$ and $\psi_{i, j}: \mathbb{F}^{n} \times\{0,1\}^{\beta_{2}} \mapsto \mathbb{F}^{n}$ such that for all $\ell \in T^{i \rightarrow j}$,

$$
f_{j}(\ell)=\psi_{i, j}\left(\ell_{i}, a_{i, j}(\ell)\right) .
$$


Proof. Given $\ell \in T^{i \rightarrow j}$, let $T^{\prime}=\left\{\ell^{*} \in T^{i \rightarrow j} \mid \ell_{i}^{*}=\ell_{i}\right\}$. Then, clearly $\left|T^{\prime}\right| \leq\left|\mathbb{F}^{n}\right|=q^{n}$. Consider a partition of $T^{\prime}$ into sets $T_{1}^{\prime}, \ldots, T_{m}^{\prime}$ according to the value of function $f_{j}$. More formally, for any $u, v \in[m]$, and any $\ell^{\prime} \in T_{u}^{\prime}, \ell^{\prime \prime} \in T_{v}^{\prime}$, we have that $f_{j}\left(\ell^{\prime}\right)=f_{j}\left(\ell^{\prime \prime}\right)$ if and only if $u=v$. By definition of $T^{i \rightarrow j}$, we have that $\left|T_{u}^{\prime}\right| \geq \frac{q^{n}}{2^{\beta_{2}}}$ for all $u \in[m]$. Thus

$$
m \leq \frac{\left|T^{\prime}\right| \cdot 2^{\beta_{2}}}{q^{n}} \leq 2^{\beta_{2}}
$$

We define $a_{i, j}(\ell)$ as the binary representation of $k$ such that $\ell \in T_{k}^{\prime}$. Now, it is easy to see that we can determine $f_{j}(\ell)$ given $\ell_{i}$ and $a_{i, j}(\ell)$.

We now define disjoint subsets $\mathcal{L}_{\text {mix }, 1}, \mathcal{L}_{\text {mix }, 2} \subseteq \mathcal{L}_{1}$ as follows.

$$
\begin{gathered}
\mathcal{L}_{\text {mix }, 1}:=\left\{\ell \in \mathcal{L}_{1} \mid \ell \notin T^{1 \rightarrow 1} \cup T^{2 \rightarrow 1}\right\}, \\
\mathcal{L}_{\text {mix }, 2}:=\left\{\ell \in \mathcal{L}_{1} \backslash \mathcal{L}_{\text {mix }, 1} \mid \ell \notin T^{1 \rightarrow 2} \cup T^{2 \rightarrow 2}\right\} .
\end{gathered}
$$

Informally speaking, $\ell \in \mathcal{L}_{\text {mix }, j}$ implies that $f_{j}(\ell)$ depends on both $\ell_{1}$ and $\ell_{2}$. Now, let

$$
\mathcal{L}_{2}:=\mathcal{L}_{1} \backslash\left(\mathcal{L}_{\text {mix }, 1} \cup \mathcal{L}_{\text {mix }, 2}\right) .
$$

We denote $\mathcal{T}(\ell, i)$ to be the set of $j \in\{1,2\}$ such that $\ell \in T^{i \rightarrow j}$. Note that by the definition of $\mathcal{L}_{\text {mix }, j}$, for any $\ell \in \mathcal{L}_{2}$ we have that $\mathcal{T}(\ell, 1) \cup \mathcal{T}(\ell, 2)=\{1,2\}$. We further partition $\mathcal{L}_{2}$ into $\mathcal{L}_{\text {id }}$, $\mathcal{L}_{\text {swap }}$, and $\mathcal{L}_{\text {rem }}$ as follows.

$$
\begin{gathered}
\mathcal{L}_{\text {id }}:=\left\{\ell \in \mathcal{L}_{2} \mid \mathcal{T}(\ell, 1)=\{1\}, \mathcal{T}(\ell, 2)=\{2\}\right\}, \\
\mathcal{L}_{\text {swap }}:=\left\{\ell \in \mathcal{L}_{2} \backslash \mathcal{L}_{\text {id }} \mid \mathcal{T}(\ell, 1)=\{2\}, \mathcal{T}(\ell, 2)=\{1\}\right\},
\end{gathered}
$$

and

$$
\mathcal{L}_{\text {rem }}:=\mathcal{L}_{2} \backslash\left(\mathcal{L}_{\text {id }} \cup \mathcal{L}_{\text {swap }}\right)
$$

The partitioning of $\mathcal{R}$ is defined similarly. We will later consider the partitions of $\mathcal{L} \times \mathcal{R}$ to be the product of individual partitions of $\mathcal{L}$ and $\mathcal{R}$ (hence, at the end $\mathcal{L} \times \mathcal{R}$ is partitioned into $7^{2}$ parts).

\subsection{Analyzing the parts}

We will argue that for any part, either its probability is small, or $\phi_{f, g}(L, R)$ conditioned on $(L, R)$ belonging to it, is close to some distribution in $\mathcal{D}$. We then apply Lemma 8.1 to obtain a proof of Theorem 5.1 ,

\subsubsection{Case: " $f$ or $g$ is far from bijection"}

Lemma 8.4. For $i=1,2$, and $\mathcal{R}^{*} \subset \mathcal{R}$, if $\left|\mathcal{L}_{\mathrm{ffb}, i} \times \mathcal{R}^{*}\right| \geq q^{4 \tau n} \cdot 2^{-s}$ then there exists a distribution $D$ that is a convex combination of distributions in $\mathcal{D}_{\text {forget }, i}$ such that

$$
\Delta\left(\left.\phi_{f, g}(L, R)\right|_{(L, R) \in \mathcal{L}_{\mathrm{ffb}, i} \times \mathcal{R}^{*}} ; D\right) \leq 2^{-s} .
$$

Proof. Without loss of generality, let $i=1$, and let $\left|\mathcal{L}_{\mathrm{ffb}, 1} \times \mathcal{R}\right| \geq q^{4 \tau n} \cdot 2^{-s}$. Let $\tilde{L}, \tilde{R}$ be distributed uniformly over $\mathcal{L}_{\text {ffb }, 1}$ and $\mathcal{R}^{*}$ respectively. Note that by the assumption we have that $\left|\mathcal{L}_{\text {ffb }, 1}\right| \geq$ $q^{2 \tau n} \cdot 2^{-s}$ and $\left|\mathcal{R}^{*}\right| \geq q^{2 \tau n} \cdot 2^{-s}$. Thus,

$$
\mathbf{H}_{\infty}\left(\tilde{L}_{1}\right) \geq(2 \tau-1) n \log q-s \quad \text { and } \quad \mathbf{H}_{\infty}\left(\tilde{L}_{2} \mid \tilde{L}_{1}\right) \geq(2 \tau-1) n \log q-s
$$


and

$$
\mathbf{H}_{\infty}\left(\tilde{R}_{1}\right) \geq(2 \tau-1) n \log q-s \quad \text { and } \quad \mathbf{H}_{\infty}\left(\tilde{R}_{2} \mid \tilde{R}_{1}\right) \geq(2 \tau-1) n \log q-s .
$$

Denote $\left\langle\tilde{L}_{k}, \tilde{R}_{k}\right\rangle$ by $X_{k}$, and $\left\langle f_{k}(\tilde{L}), g_{k}(\tilde{R})\right\rangle$ by $X_{k}^{\prime}$ for $k=1,2$. We have that

$$
\mathbf{H}_{\infty}\left(\tilde{L}_{1} \mid X_{2}, f(\tilde{L})\right) \geq \frac{\beta_{1}}{2}-\log q .
$$

Also, using Lemma 2.5, we get that $\tilde{L}$ and $\tilde{R}$ (and hence $\tilde{L}_{1}$ and $\tilde{R}_{1}$ ) are independent given $f(\tilde{L})$, $\tilde{R}_{2}$, and $X_{2}$. Thus, using the fact that Ext is a strong two-source extractor, we have that

$$
X_{1}, \tilde{R}_{1}, X_{2}, f(\tilde{L}), \tilde{R}_{2} \approx_{2^{-(s+1)}} U_{1}, \tilde{R}_{1}, X_{2}, f(\tilde{L}), \tilde{R}_{2},
$$

where $U_{1}$ is uniformly random in $\mathbb{F}$. This implies that

$$
X_{1}, X_{2}, X_{1}^{\prime}, X_{2}^{\prime} \approx_{2^{-(s+1)}} U_{1}, X_{2}, X_{1}^{\prime}, X_{2}^{\prime} .
$$

Now, $X_{1}^{\prime}, X_{2}^{\prime}$ are independent of $U_{1}$, and can be seen as a randomized function of $X_{2}$. Let

$$
U_{1}, X_{2}, X_{1}^{\prime}, X_{2}^{\prime} \equiv U_{1}, X_{2}, h_{1}\left(X_{2}, Z\right), h_{2}\left(X_{2}, Z\right),
$$

for $Z$ independent of $U_{1}, X_{2}$. Using Equation 8.5 and 8.6, and that Ext is a two-source randomness extractor, we have that $X_{2}$ is $2^{-(s+1)}$-close to uniform. Thus, using triangle inequality, we get that

$$
X_{1}, X_{2}, X_{1}^{\prime}, X_{2}^{\prime} \approx_{2^{-s}} U_{1}, U_{2}, h_{1}\left(U_{2}, Z\right), h_{2}\left(U_{2}, Z\right)
$$

which implies the result.

In a symetric way we can prove the following.

Lemma 8.8. For $i=1,2$, and $\mathcal{L}^{*} \subset \mathcal{L}$, if $\left|\mathcal{L}^{*} \times \mathcal{R}_{\mathrm{ffb}, i}\right| \geq q^{4 \tau n} \cdot 2^{-s}$ then there exists a distribution $D$ that is a convex combination of distributions in $\mathcal{D}_{\text {forget }, i}$ such that

$$
\Delta\left(\left.\phi_{f, g}(L, R)\right|_{(L, R) \in \mathcal{L}^{*} \times \mathcal{R}_{\mathrm{ffb}, i}} ; D\right) \leq 2^{-s} .
$$

\subsubsection{Case: "output of $f$ or $g$ is mixed"}

Lemma 8.9. For $j=1,2$, and $\mathcal{R}^{*} \subset \mathcal{R}$, if $\left|\mathcal{L}_{\text {mix }, j} \times \mathcal{R}^{*}\right| \geq q^{4 \tau n} \cdot 2^{-s}$ then there exists a distribution $D$ that is a convex combination of distributions in $\mathcal{D}_{\text {unif }, j}$, such that

$$
\Delta\left(\left.\phi_{f, g}(L, R)\right|_{(L, R) \in \mathcal{L}_{\text {mix }, j} \times \mathcal{R}^{*}} ; D\right) \leq 2^{-s} .
$$

Proof. Let us assume that $\left|\mathcal{L}_{\text {mix }, 1} \times \mathcal{R}^{*}\right| \geq q^{4 \tau n} \cdot 2^{-s}$. (Case $j=2$ is analogous.) From the assumptions we have that $\left|\mathcal{L}_{\text {mix,1 }}\right| \geq q^{2 \tau n} \cdot 2^{-s}$ and $\left|\mathcal{R}^{*}\right| \geq q^{2 \tau n} \cdot 2^{-s}$. Let $\tilde{L}, \tilde{R}$ be distributed uniformly over $\mathcal{L}_{\text {mix }, 1}$ and $\mathcal{R}^{*}$ respectively. Denote $\operatorname{Ext}\left(\tilde{L}_{k}, \tilde{R}_{k}\right)$ by $X_{k}$, and $\left\langle f_{k}(\tilde{L}), g_{k}(\tilde{R})\right\rangle$ by $X_{k}^{\prime}$ for $k=1,2$. Reasoning similarly as in Lemma 8.4, we have that $X_{1}$ is $q^{-3} \cdot 2^{-(s+1)}$-close to uniform, and also $X_{2}$ is $q^{-3} \cdot 2^{-(s+1)}$-close to uniform given $\tilde{L}_{1}, \tilde{R}_{1}$, and hence using the hybrid argument, we have that

$$
X_{1}, X_{2} \approx_{2^{-s} q^{-3}} U_{1}, U_{2}
$$


where $U_{1}, U_{2}$, are independent and uniformly distributed in $\mathbb{F}$. We now give a lower bound for $\mathbf{H}_{\infty}\left(\tilde{L}_{i} \mid f_{1}(\tilde{L})\right)$ for $i=1,2$ using the definition of $\mathcal{L}_{\text {mix }, 1}$.

$$
\begin{aligned}
\mathbf{H}_{\infty}\left(\tilde{L}_{i} \mid f_{j}(\tilde{L})\right) & =-\log \left(\sum_{y \in \mathbb{F}^{n}} \max _{\ell_{i} \in \mathbb{F}^{n}} \operatorname{Pr}\left(\tilde{L}_{i}=\ell_{i} \wedge f_{j}(\tilde{L})=y\right)\right) \\
& \geq-\log \left(\sum_{y \in \mathbb{F}^{n}} \frac{q^{n} 2^{-\beta_{2}}}{\left|\mathcal{L}_{\text {mix }, 1}\right|}\right) \\
& \geq-\log \left(\frac{q^{2 n} 2^{-\beta_{2}}}{q^{2 \tau n} 2^{-s}}\right) \geq \beta_{2}-2(1-\tau) n \log q-s .
\end{aligned}
$$

Thus, we have that for $i=1,2, X_{i}$ is $2^{-s-1} q^{-3}$-close to uniform given $f_{1}(\tilde{L}), \tilde{R}$, and hence,

$$
\Delta\left(X_{i}, \operatorname{Ext}\left(f_{1}(\tilde{L}), g_{1}(\tilde{R})\right) ; U_{i}, \operatorname{Ext}\left(f_{1}(\tilde{L}), g_{1}(\tilde{R})\right)\right) \leq q^{-3} 2^{-s-1}
$$

Also, since $\mathcal{L}_{\text {mix, } 1}$ and $\mathcal{R}^{*}$ are in the complement of $\mathcal{L}_{\text {ffb }}$, and $\mathcal{R}_{\text {ffb }}$, respectively, we have that

$$
\mathbf{H}_{\infty}\left(f_{j}(\tilde{L})\right) \geq \mathbf{H}_{\infty}(f(\tilde{L}))-n \log q \geq(2 \tau-1) n \log q-\beta_{1}-s,
$$

and

$$
\mathbf{H}_{\infty}\left(g_{j}(\tilde{R})\right) \geq(2 \tau-1) n \log q-\beta_{1}-s
$$

This implies that

$$
\Delta\left(X_{i}, X_{1}^{\prime} ; U_{i}, U_{1}^{\prime}\right) \leq q^{-3} \cdot 2^{-s},
$$

where $U_{1}^{\prime}$ is uniform in $\mathbb{F}$.

Now, we claim that

$$
\Delta\left(X_{1}, X_{2}, X_{1}^{\prime} ; U_{1}, U_{2}, U_{1}^{\prime}\right) \leq 2^{-s} .
$$

If not, then by the XOR Lemma, there exist $a_{1}, a_{2}, a_{3}$, not all zero such that $a_{1} X_{1}+a_{2} X_{2}+a_{3} X_{1}^{\prime}$ is not $2^{-s} \cdot q^{-3}$ close to uniform. By Equation 8.10, we have that $a_{3} \neq 0$, and by equation 8.11, we have that $a_{1}, a_{2} \neq 0$. Consider two sources in $\mathbb{F}^{3}$ as $\left(a_{1} \tilde{L}_{1}, a_{2} \tilde{L}_{2}, a_{3} f_{1}(\tilde{L})\right)$ and $\left(\tilde{R}_{1}, \tilde{R}_{2}, g_{1}(\tilde{R})\right)$. Applying Ext to these two sources gives $a_{1} X_{1}+a_{2} X_{2}+a_{3} X_{1}^{\prime}$. The two sources have min-entropy at least $2 \tau n \log q-s$, and hence $\sum_{i=1}^{t} a_{i} X_{i}+a_{t+1} \operatorname{Ext}\left(f_{j}(\tilde{L}), g_{j}(\tilde{R})\right)$ is $2^{-s} q^{-3}$-close to uniform, which is a contradiction.

Symmetrically, we get that

Lemma 8.13. For $j=1,2$, and $\mathcal{L}^{*} \subset \mathcal{L}$, if $\left|\mathcal{L}^{*} \times \mathcal{R}_{\text {mix }, j}\right| \geq q^{4 \tau n} \cdot 2^{-s}$ then

$$
\Delta\left(\left.\phi_{f, g}(L, R)\right|_{(L, R) \in \mathcal{L}^{*} \times \mathcal{R}_{\text {mix }, j}} ; D\right) \leq 2^{-s},
$$

for some $D$ that is a convex combination of distributions in $\mathcal{D}_{\text {unif }, j}$.

8.3.3 Case " $\tilde{L} \in \mathcal{L}_{\text {id }} \cup \mathcal{L}_{\text {swap }}$ and $\tilde{R} \in \mathcal{R}_{\text {id }} \cup \mathcal{R}_{\text {swap" }}$ "

Lemma 8.14. If $\left|\mathcal{L}_{\text {id }} \times \mathcal{R}_{\text {swap }}\right| \geq q^{4 \tau n} \cdot 2^{-s}$ then there exists a distribution $D$ that is a convex combination of distributions in $\mathcal{D}_{\text {forget }, 1}$ such that

$$
\Delta\left(\left.\phi_{f, g}(L, R)\right|_{(L, R) \in \mathcal{L}_{\text {id }} \times \mathcal{R}_{\text {swap }}} ; D\right) \leq 2^{-s} .
$$


Proof. This proof is almost identical to that of Lemma 8.4, except that it makes crucial use of Lemma 8.3. Let $\left|\mathcal{L}_{\text {id }} \times \mathcal{R}_{\text {swap }}\right| \geq q^{4 \tau n} \cdot 2^{-s}$. Let $\tilde{L}, \tilde{R}$ be distributed uniformly over $\mathcal{L}_{\text {id }}$ and $\mathcal{R}_{\text {swap }}$ respectively. Note that by the assumption we have that $\left|\mathcal{L}_{\text {id }}\right| \geq q^{2 \tau n} \cdot 2^{-s}$ and $\left|\mathcal{R}_{\text {swap }}\right| \geq q^{2 \tau n} \cdot 2^{-s}$. Thus, for $k=1,2$,

$$
\mathbf{H}_{\infty}\left(\tilde{L}_{k} \mid \tilde{L}_{k-1}\right) \geq(2 \tau-1) n \log q-s,
$$

and

$$
\mathbf{H}_{\infty}\left(\tilde{R}_{k} \mid \tilde{R}_{k-1}\right) \geq(2 \tau-1) n \log q-s,
$$

where $\tilde{L}_{0}=\tilde{R}_{0} \equiv 0$. Denote $\left\langle\tilde{L}_{k}, \tilde{R}_{k}\right\rangle$ by $X_{k}$, and $\left\langle f_{k}(\tilde{L}), g_{k}(\tilde{R})\right\rangle$ by $X_{k}^{\prime}$ for $k=1,2$. By Lemma 8.3 there exists maps $a_{1,1}, a_{2,2}$ from $\mathcal{L}_{\text {id }}$ to $\{0,1\}^{\beta_{2}}$, and $b_{1,2}, b_{2,1}$ from $\mathcal{R}_{\text {swap }}$ to $\{0,1\}^{\beta_{2}}$, such that $f_{1}(\tilde{L}), f_{2}(\tilde{L}), g_{1}(\tilde{R}), g_{2}(\tilde{R})$ are determined uniquely given $\left(\tilde{L}_{1}, a_{1,1}(\tilde{L})\right),\left(\tilde{L}_{2}, a_{2,2}(\tilde{L})\right),\left(\tilde{R}_{2}, b_{2,1}(\tilde{R})\right)$, $\left(\tilde{R}_{1}, b_{1,2}(\tilde{R})\right)$, respectively.

Also, using Lemma 2.5 , we get that $\tilde{L}$ and $\tilde{R}$ (and hence $\tilde{L}_{1}$ and $\tilde{R}_{1}$ ) are independent given $X_{2}=\left\langle\tilde{L}_{2}, \tilde{R}_{2}\right\rangle, X_{1}^{\prime}$, and $X_{2}^{\prime}$. Thus, using the fact that Ext is a strong two-source extractor, we have that

$$
X_{1}, \tilde{R}_{1}, X_{2}, f(\tilde{L}), \tilde{R}_{2} \approx_{2^{-(s+1)}} U_{1}, \tilde{R}_{1}, X_{2}, f(\tilde{L}), \tilde{R}_{2},
$$

where $U_{1}$ is uniformly random in $\mathbb{F}$. This implies that

$$
X_{1}, X_{2}, X_{1}^{\prime}, X_{2}^{\prime} \approx_{2^{-(s+1)}} U_{1}, X_{2}, X_{1}^{\prime}, X_{2}^{\prime}
$$

Now, $X_{1}^{\prime}, X_{2}^{\prime}$ are independent of $U_{1}$, and can be seen as a randomized function of $X_{2}$. Let

$$
U_{1}, X_{2}, X_{1}^{\prime}, X_{2}^{\prime} \equiv U_{1}, X_{2}, h_{1}\left(X_{2}, Z\right), h_{2}\left(X_{2}, Z\right),
$$

for $Z$ independent of $U_{1}, X_{2}$. Using Equation 8.15 and 8.16, and that Ext is a two-source randomness extractor, we have that $X_{2}$ is $2^{-(s+1)}$-close to uniform. Thus, using equation triangle inequality, we get that

$$
X_{1}, X_{2}, X_{1}^{\prime}, X_{2}^{\prime} \approx_{2^{-s}} U_{1}, U_{2}, h_{1}\left(U_{2}, Z\right), h_{2}\left(U_{2}, Z\right),
$$

which implies the result.

Symmetrically, we get the following:

Lemma 8.17. If $\left|\mathcal{L}_{\text {swap }} \times \mathcal{R}_{\text {id }}\right| \geq q^{4 \tau n} \cdot 2^{-s}$ then there exists a distribution $D$ that is a convex combination of distributions in $\mathcal{D}_{\text {forget }, 1}$ such that

$$
\Delta\left(\left.\phi_{f, g}(L, R)\right|_{(L, R) \in \mathcal{L}_{\text {swap }} \times \mathcal{R}_{\text {id }}} ; D\right) \leq 2^{-s} .
$$

We now look at the case when $L, R$ are restricted to $\mathcal{L}_{\text {id }}$, and $\mathcal{R}_{\text {id }}$, respectively.

Lemma 8.18. If $\left|\mathcal{L}_{\mathrm{id}} \times \mathcal{R}_{\mathrm{id}}\right| \geq q^{4 \tau n} \cdot 2^{-s}$ then there exists a distribution $D$ that is a convex combination of distributions in $\mathcal{D}_{\text {id }}$ such that

$$
\Delta\left(\left.\phi_{f, g}(L, R)\right|_{(L, R) \in \mathcal{L}_{\text {id }} \times \mathcal{R}_{\text {id }}} ; D\right) \leq 2^{-s} .
$$

Proof. Let $\left|\mathcal{L}_{\text {id }} \times \mathcal{R}_{\text {id }}\right| \geq q^{4 \tau n} \cdot 2^{-s}$. Let $\tilde{L}, \tilde{R}$ be distributed uniformly over $\mathcal{L}_{\text {id }}$ and $\mathcal{R}_{\text {id }}$ respectively. Note that by the assumption we have that $\left|\mathcal{L}_{\text {id }}\right| \geq q^{2 \tau n} \cdot 2^{-s}$ and $\left|\mathcal{R}_{\text {swap }}\right| \geq q^{2 \tau n} \cdot 2^{-s}$. Denote $\left\langle\tilde{L}_{k}, \tilde{R}_{k}\right\rangle$ by $X_{k}$, and $\left\langle f_{k}(\tilde{L}), g_{k}(\tilde{R})\right\rangle$ by $X_{k}^{\prime}$ for $k=1,2$.

By Lemma 8.3 there exists maps $a_{1,1}, a_{2,2}$ from $\mathcal{L}_{\text {id }}$ to $\{0,1\}^{\beta_{2}}$, and $b_{1,1}, b_{2,2}$ from $\mathcal{R}_{\text {id }}$ to $\{0,1\}^{\beta_{2}}$, such that $f_{1}(\tilde{L}), f_{2}(\tilde{L}), g_{1}(\tilde{R}), g_{2}(\tilde{R})$ are determined uniquely given $\left(\tilde{L}_{1}, a_{1,1}(\tilde{L})\right),\left(\tilde{L}_{2}, a_{2,2}(\tilde{L})\right)$, $\left(\tilde{R}_{1}, b_{1,1}(\tilde{R})\right),\left(\tilde{R}_{2}, b_{2,2}(\tilde{R})\right)$, respectively. We define the random variable $Y$ as

$$
Y:=\tilde{L}_{1}, \quad \tilde{R}_{2}, \quad a_{1,1}(\tilde{L}), \quad a_{2,2}(\tilde{L}), \quad b_{1,1}(\tilde{R}), \quad b_{2,2}(\tilde{R}) .
$$


Note that $X_{1}^{\prime}$ is a deterministic function of $Y$ and $\tilde{R}_{1}$. Similarly, $X_{2}^{\prime}$ is a deterministic function of $Y$ and $\tilde{L}_{2}$. Let $W_{1}$ be independent randomness used to sample $\tilde{R}_{1}$ given $Y$ and $X_{1}$, and let $W_{2}$ be independent randomness used to sample $\tilde{L}_{2}$ given $Y$ and $X_{2}$. Note that $W_{1}, W_{2}$ are independent from each other and from $X_{1}, X_{2}, Y$. Therefore, we have that

$$
X_{1}, X_{2}, X_{1}^{\prime}, X_{2}^{\prime} \equiv X_{1}, X_{2}, h_{1}\left(X_{1}, Y, W_{1}\right), h_{2}\left(X_{2}, Y, W_{2}\right),
$$

for some functions $h_{1}, h_{2}$. Also, using Lemma 2.3 , we have that

$$
\begin{array}{rcc}
X_{1}, X_{2}, Y, W_{1}, W_{2} & \approx_{2^{-(s+1)}} & U_{1}, X_{2}, Y, W_{1}, W_{2} \\
& \approx_{2^{-(s+1)}} & U_{1}, U_{2}, Y, W_{1}, W_{2}
\end{array}
$$

This implies the desired result using equation 8.19 , and Lemma 2.1.

Symmetrically, we get the following:

Lemma 8.20. If $\left|\mathcal{L}_{\text {swap }} \times \mathcal{R}_{\text {swap }}\right| \geq q^{4 \tau n} \cdot 2^{-s}$ then there exists a distribution $D$ that is a convex combination of distributions in $\mathcal{D}_{\text {swap }}$ such that

$$
\Delta\left(\left.\phi_{f, g}(L, R)\right|_{(L, R) \in \mathcal{L}_{\text {swap }} \times \mathcal{R}_{\text {swap }}} ; D\right) \leq 2^{-s} .
$$

\subsubsection{Remaining cases}

Lemma 8.21. $\left|\mathcal{L}_{\text {rem }}\right| \leq q^{2 \tau n} 2^{-s}$, and $\left|\mathcal{R}_{\text {rem }}\right| \leq q^{2 \tau n} 2^{-s}$

Proof. Consider any $\ell \in \mathcal{L}_{\text {rem }}$. Since $\ell \notin \mathcal{L}_{\text {mix }, j}$, we have that $\mathcal{T}(\ell, 1) \cup \mathcal{T}(\ell, 2)=\{1,2\}$. Also, since $\ell \notin \mathcal{L}_{\text {id }} \cup \mathcal{L}_{\text {swap }}$, there exists some $k \in\{1,2\}$, such that $\mathcal{T}(\ell, k)=\{1,2\}$. Thus, using Lemma 8.3 . we have that $f(\ell)$ can be determined given $\ell_{k}, a_{k, 1}, a_{k, 2}$. This implies that $f(\ell)$ can be determined given at most $\tau n \log q+2 \beta_{2}$ bits. Therefore

$$
\left|\mathcal{L}_{\text {rem }}\right| \leq q^{\tau n} 2^{2 \beta_{2}} \leq q^{2 \tau n} 2^{-s} .
$$

Symmetrically, $\left|\mathcal{R}_{\text {rem }}\right| \leq q^{2 \tau n} 2^{-s}$.

\subsection{Finishing the proof}

We partitioned $\mathcal{L} \times \mathcal{R}$ into following cases.

- $\mathcal{L}_{\mathrm{ffb}, i} \times \mathcal{R}^{*}($ see Lemma 8.4 )

- $\mathcal{L}^{*} \times \mathcal{R}_{\mathrm{ffb}, i}$ (see Lemma 8.8)

- $\mathcal{L}_{\text {mix }, j} \times \mathcal{R}^{*}$ (see Lemma 8.9 p

- $\mathcal{L}^{*} \times \mathcal{R}_{\text {mix }, j}$ (see Lemma 8.13)

- $\mathcal{L}_{\text {id }} \times \mathcal{R}_{\text {swap }}$ (see Lemma 8.14)
- $\mathcal{L}_{\text {swap }} \times \mathcal{R}_{\text {id }}($ see Lemma 8.17)

- $\mathcal{L}_{\text {id }} \times \mathcal{R}_{\text {id }}$ (see Lemma 8.18)

- $\mathcal{L}_{\text {swap }} \times \mathcal{R}_{\text {swap }}($ see Lemma 8.20

- $\mathcal{L}_{\text {rem }} \times \mathcal{R}^{*}$ and $\mathcal{L}^{*} \times \mathcal{R}_{\text {rem }}$ (see Lemma 8.21)

We showed that in every case for partition $\mathcal{L}^{*} \times \mathcal{R}^{*}$ we get either $\frac{\left|\mathcal{L}^{*} \times \mathcal{R}^{*}\right|}{|\mathcal{L} \times \mathcal{R}|} \leq 2^{-s}$ or there exists $D^{\prime}$ from $\mathcal{D}$ such that

$$
\Delta\left(\left.\phi_{f, g}(L, R)\right|_{L, R \in \mathcal{L}^{*} \times \mathcal{R}^{*}} ; D^{\prime}\right) \leq 2^{-s},
$$


where $\mathcal{D}$ is a convex combination of distributions $\mathcal{D}_{\text {id }} \cup \mathcal{D}_{\text {swap }} \cup \mathcal{D}_{\text {unif }, 1} \cup \mathcal{D}_{\text {unif }, 2} \cup \mathcal{D}_{\text {forget }, 1} \cup \mathcal{D}_{\text {forget }, 2}$. We partitioned both $\mathcal{L}$ and $\mathcal{R}$ each into 7 subsets, thus by Lemma 8.1 we obtain that there exists a distribution $D$ in $\mathcal{D}$ such that

$$
\Delta\left(\phi_{f, g}(L, R) ; D\right) \leq 7^{2} \cdot 2^{-s} .
$$

This finishes the proof.

\section{Conclusions and Open Problems}

Our main result is a generic transformation from non-malleable codes in the 2-split-state model to non-malleable codes in the 2 -split-state model that is resilient to leakage of length upto 1/12-th of the length of the codeword. Combining with the best known non-malleable codes in the 2-split state model achieved by a subsequent work [ADKO14], we get constant-rate 1/12-leakage resilient non-malleable codes.

We also observe in Section 7 that the result of [CG14b] implies that we can achieve non-malleable codes resilient to a fraction of leakage arbitrarily close to 1 .

Thus, our work can be viewed as initiating the study and achieving a constant factor leakage resilience for information-theoretically secure non-malleable codes. In view of the existential result, the main open question is whether we can give an efficient construction of non-malleable codes that can achieve leakage-resilience larger than a 1/12-th fraction, and thereby make non-malleable codes practically more useful.

\section{References}

[ADKO14] Divesh Aggarwal, Yevgeniy Dodis, Tomasz Kazana, and Maciej Obremski. Nonmalleable reductions and applications, 2014. Unpublished manuscript.

[ADL14] Divesh Aggarwal, Yevgeniy Dodis, and Shachar Lovett. Non-malleable codes from additive combinatorics. In David B. Shmoys, editor, 46th ACM STOC, pages 774783, New York, NY, USA, May 31 - June 3, 2014. ACM Press.

[Agg14] Divesh Aggarwal. Affine-evasive sets modulo a prime. Cryptology ePrint Archive, Report 2014/328, 2014. http://eprint.iacr.org/.

[AK96] Ross Anderson and Markus Kuhn. Tamper resistance - a cautionary note. In The Second USENIX Workshop on Electronic Commerce, pages 1-11, November 1996.

[BDL14] Nir Bitansky, Dana Dachman-Soled, and Huijia Lin. Leakage-tolerant computation with input-independent preprocessing. In Juan A. Garay and Rosario Gennaro, editors, CRYPTO 2014, Part II, volume 8617 of LNCS, pages 146-163, Santa Barbara, CA, USA, August 17-21, 2014. Springer, Berlin, Germany.

[BK03] Mihir Bellare and Tadayoshi Kohno. A theoretical treatment of related-key attacks: RKA-PRPs, RKA-PRFs, and applications. In Eli Biham, editor, Advances in Cryptology-EUROCRYPT 2003, volume 2656 of LNCS. Springer-Verlag, 2003. Full version available at http://www-cse.ucsd.edu/users/tkohno/papers/RKA/.

[BR93] Mihir Bellare and Phillip Rogaway. Random oracles are practical: A paradigm for designing efficient protocols. In V. Ashby, editor, ACM CCS 93, pages 62-73, Fairfax, Virginia, USA, November 3-5, 1993. ACM Press. 
[CCP12] H. Chabanne, G. Cohen, and A Patey. Secure network coding and non-malleable codes: Protection against linear tampering. In Information Theory Proceedings (ISIT), 2012 IEEE International Symposium on, pages 2546-2550, July 2012.

$\left[\mathrm{CDF}^{+} 08\right]$ Ronald Cramer, Yevgeniy Dodis, Serge Fehr, Carles Padró, and Daniel Wichs. Detection of algebraic manipulation with applications to robust secret sharing and fuzzy extractors. In Nigel P. Smart, editor, EUROCRYPT 2008, volume 4965 of LNCS, pages 471-488, Istanbul, Turkey, April 13-17, 2008. Springer, Berlin, Germany.

[CG88] B. Chor and O. Goldreich. Unbiased bits from sources of weak randomness and probabilistic communication complexity. SIAM Journal on Computing, 17(2):230261, 1988.

[CG14a] Mahdi Cheraghchi and Venkatesan Guruswami. Capacity of non-malleable codes. In Moni Naor, editor, ITCS 2014, pages 155-168, Princeton, NJ, USA, January 12-14, 2014. ACM.

[CG14b] Mahdi Cheraghchi and Venkatesan Guruswami. Non-malleable coding against bit-wise and split-state tampering. In Theory of Cryptography - 11th Theory of Cryptography Conference, TCC 2014, San Diego, CA, USA, February 24-26, 2014. Proceedings, pages 440-464, 2014.

[CMTV14] Sandro Coretti, Ueli Maurer, Björn Tackmann, and Daniele Venturi. From single-bit to multi-bit public-key encryption via non-malleable codes. Cryptology ePrint Archive, Report 2014/324, 2014. http://eprint.iacr.org/.

[CZ14] Eshan Chattopadhyay and David Zuckerman. Non-malleable codes against constant split-state tampering. In FOCS, 2014.

[DDN98] Danny Dolev, Cynthia Dwork, and Moni Naor. Non-malleable cryptography, 1998. Manuscript.

[DDV10] Francesco Davì, Stefan Dziembowski, and Daniele Venturi. Leakage-resilient storage. In Juan A. Garay and Roberto De Prisco, editors, SCN, volume 6280 of Lecture Notes in Computer Science, pages 121-137. Springer, 2010.

[DF12] Stefan Dziembowski and Sebastian Faust. Leakage-resilient circuits without computational assumptions. In Ronald Cramer, editor, TCC 2012, volume 7194 of LNCS, pages 230-247, Taormina, Sicily, Italy, March 19-21, 2012. Springer, Berlin, Germany.

[DK12] Dana Dachman-Soled and Yael Tauman Kalai. Securing circuits against constantrate tampering. In Reihaneh Safavi-Naini and Ran Canetti, editors, CRYPTO 2012, volume 7417 of LNCS, pages 533-551, Santa Barbara, CA, USA, August 19-23, 2012. Springer, Berlin, Germany.

[DKO13] Stefan Dziembowski, Tomasz Kazana, and Maciej Obremski. Non-malleable codes from two-source extractors. In Ran Canetti and Juan A. Garay, editors, CRYPTO 2013, Part II, volume 8043 of LNCS, pages 239-257, Santa Barbara, CA, USA, August 18-22, 2013. Springer, Berlin, Germany.

[DORS08] Yevgeniy Dodis, Rafail Ostrovsky, Leonid Reyzin, and Adam Smith. Fuzzy extractors: How to generate strong keys from biometrics and other noisy data. SIAM J. Comput., 38(1):97-139, March 2008. 
[DP07] Stefan Dziembowski and Krzysztof Pietrzak. Intrusion-resilient secret sharing. In FOCS, pages 227-237. IEEE Computer Society, 2007.

[DP08] Stefan Dziembowski and Krzysztof Pietrzak. Leakage-resilient cryptography. In 49th Symposium on Foundations of Computer Science, pages 293-302, Philadelphia, PA, USA, October 25-28 2008. IEEE Computer Society.

[DPW10] Stefan Dziembowski, Krzysztof Pietrzak, and Daniel Wichs. Non-malleable codes. In Andrew Chi-Chih Yao, editor, ICS 2010, pages 434-452, Tsinghua University, Beijing, China, January 5-7, 2010. Tsinghua University Press.

[ECR] ECRYPT. Side channel cryptanalysis lounge. last accessed: August 26, 2009. http: //www.crypto.ruhr-uni-bochum.de/en_sclounge.html.

[FKPR10] Sebastian Faust, Eike Kiltz, Krzysztof Pietrzak, and Guy N. Rothblum. Leakageresilient signatures. In Daniele Micciancio, editor, TCC 2010, volume 5978 of LNCS, pages 343-360, Zurich, Switzerland, February 9-11, 2010. Springer, Berlin, Germany.

[FMNV14a] S. Faust, P. Mukherjee, J. Nielsen, and D. Venturi. Continuous non-malleable codes. In Theory of Cryptography Conference - TCC. Springer, 2014.

[FMNV14b] Sebastian Faust, Pratyay Mukherjee, Jesper Buus Nielsen, and Daniele Venturi. A tamper and leakage resilient random access machine. Cryptology ePrint Archive, Report 2014/338, 2014. http://eprint.iacr.org/2014/338.

[FMVW14] Sebastian Faust, Pratyay Mukherjee, Daniele Venturi, and Daniel Wichs. Efficient non-malleable codes and key-derivation for poly-size tampering circuits. In Phong Q. Nguyen and Elisabeth Oswald, editors, EUROCRYPT 2014, volume 8441 of LNCS, pages 111-128, Copenhagen, Denmark, May 11-15, 2014. Springer, Berlin, Germany.

[FPV11] Sebastian Faust, Krzysztof Pietrzak, and Daniele Venturi. Tamper-proof circuits: How to trade leakage for tamper-resilience. In Luca Aceto, Monika Henzinger, and Ji Sgall, editors, Automata, Languages and Programming, volume 6755 of Lecture Notes in Computer Science, pages 391-402. Springer Berlin Heidelberg, 2011.

$\left[\mathrm{GLM}^{+} 04\right]$ Rosario Gennaro, Anna Lysyanskaya, Tal Malkin, Silvio Micali, and Tal Rabin. Algorithmic tamper-proof (ATP) security: Theoretical foundations for security against hardware tampering. In Moni Naor, editor, TCC 2004, volume 2951 of LNCS, pages 258-277, Cambridge, MA, USA, February 19-21, 2004. Springer, Berlin, Germany.

[GR12] Shafi Goldwasser and Guy N. Rothblum. How to compute in the presence of leakage. In 53rd FOCS, pages 31-40, New Brunswick, NJ, USA, October 20-23, 2012. IEEE Computer Society Press.

[IPSW06] Yuval Ishai, Manoj Prabhakaran, Amit Sahai, and David Wagner. Private circuits II: Keeping secrets in tamperable circuits. In Serge Vaudenay, editor, Advances in Cryptology-EUROCRYPT 2006, volume 4004 of LNCS, pages 308-327. SpringerVerlag, 2006.

[ISW03] Yuval Ishai, Amit Sahai, and David Wagner. Private circuits: Securing hardware against probing attacks. In Dan Boneh, editor, Advances in CryptologyCRYPTO 2003, volume 2729 of LNCS. Springer-Verlag, 2003. 
[KKS11] Yael Tauman Kalai, Bhavana Kanukurthi, and Amit Sahai. Cryptography with tamperable and leaky memory. In Phillip Rogaway, editor, CRYPTO 2011, volume 6841 of LNCS, pages 373-390, Santa Barbara, CA, USA, August 14-18, 2011. Springer, Berlin, Germany.

[LL10] Feng-Hao Liu and Anna Lysyanskaya. Algorithmic tamper-proof security under probing attacks. In Juan A. Garay and Roberto De Prisco, editors, SCN 10, volume 6280 of LNCS, pages 106-120, Amalfi, Italy, September 13-15, 2010. Springer, Berlin, Germany.

[LL12] Feng-Hao Liu and Anna Lysyanskaya. Tamper and leakage resilience in the splitstate model. In Reihaneh Safavi-Naini and Ran Canetti, editors, CRYPTO 2012, volume 7417 of LNCS, pages 517-532, Santa Barbara, CA, USA, August 19-23, 2012. Springer, Berlin, Germany.

[MR04] Silvio Micali and Leonid Reyzin. Physically observable cryptography (extended abstract). In Moni Naor, editor, First Theory of Cryptography Conference - TCC 2004, volume 2951 of LNCS, pages 278-296. Springer-Verlag, February 19-21 2004.

[Rao07] Anup Rao. An exposition of bourgain 2-source extractor. In Electronic Colloquium on Computational Complexity (ECCC), volume 14, page 034, 2007.

\section{A Proofs of Lemmata from Section 2}

Lemma 2.4 Let $X_{1}, Y_{1} \in \mathcal{A}_{1}$, and $X_{2}, Y_{2} \in \mathcal{A}_{2}$ be random variables such that $\Delta\left(\left(X_{1}, X_{2}\right) ;\left(Y_{1}, Y_{2}\right)\right) \leq$ $\varepsilon$. Then, for any non-empty set $\mathcal{A}^{\prime} \subseteq \mathcal{A}_{1}$, we have

$$
\Delta\left(X_{2}\left|X_{1} \in \mathcal{A}^{\prime} ; Y_{2}\right| Y_{1} \in \mathcal{A}^{\prime}\right) \leq \frac{2 \varepsilon}{\operatorname{Pr}\left(X_{1} \in \mathcal{A}^{\prime}\right)} .
$$

Proof.

$$
\begin{aligned}
\Delta\left(X_{2}\left|X_{1} \in \mathcal{A}^{\prime} ; Y_{2}\right| Y_{1} \in \mathcal{A}^{\prime}\right)= & \frac{1}{2} \sum_{x \in \mathcal{A}_{2}}\left|\operatorname{Pr}\left(X_{2}=x \mid X_{1} \in \mathcal{A}^{\prime}\right)-\operatorname{Pr}\left(Y_{2}=x \mid Y_{1} \in \mathcal{A}^{\prime}\right)\right| \\
\leq & \frac{1}{2} \sum_{x \in \mathcal{A}_{2}}\left(\left|\frac{\operatorname{Pr}\left(X_{2}=x \wedge X_{1} \in \mathcal{A}^{\prime}\right)}{\operatorname{Pr}\left(X_{1} \in \mathcal{A}^{\prime}\right)}-\frac{\operatorname{Pr}\left(Y_{2}=x \wedge Y_{1} \in \mathcal{A}^{\prime}\right)}{\operatorname{Pr}\left(X_{1} \in \mathcal{A}^{\prime}\right)}\right|\right. \\
& \left.+\operatorname{Pr}\left(Y_{2}=x \wedge Y_{1} \in \mathcal{A}^{\prime}\right)\left|\frac{1}{\operatorname{Pr}\left(Y_{1} \in \mathcal{A}^{\prime}\right)}-\frac{1}{\operatorname{Pr}\left(X_{1} \in \mathcal{A}^{\prime}\right)}\right|\right) \\
\leq & \frac{\varepsilon \cdot \sum_{x \in \mathcal{A}_{2}} \operatorname{Pr}\left(Y_{1} \in \mathcal{A}^{\prime} \wedge Y_{2}=x\right)}{\operatorname{Pr}\left(Y_{1} \in \mathcal{A}^{\prime}\right) \cdot \operatorname{Pr}\left(X_{1} \in \mathcal{A}^{\prime}\right)} \\
= & \frac{2 \varepsilon}{\operatorname{Pr}\left(X_{1} \in \mathcal{A}^{\prime}\right)}+\frac{\left.\mathcal{A}^{\prime}\right)}{}
\end{aligned}
$$

Lemma 2.6 Let $X=\left(X_{1}, \ldots, X_{t}\right) \in \mathbb{F}^{t}$ be a random variable, where $\mathbb{F}$ is a finite field of order $q$. Assume that for all $a_{1}, \ldots, a_{t} \in \mathbb{F}^{t}$ not all zero, $\Delta\left(\sum_{i=1}^{t} a_{i} X_{i} ; U\right) \leq \varepsilon$, where $U$ is uniform in $\mathbb{F}$. Then $\Delta\left(X_{1}, \ldots, X_{t} ; U_{1}, \ldots, U_{t}\right) \leq \varepsilon q^{(t+2) / 2}$, where $U_{1}, \ldots, U_{t}$ are independent and uniform in $\mathbb{F}^{t}$. 
Proof. The proof uses basic Fourier analysis. Assume $\mathbb{F}$ has characteristic $p$. Let $\omega=e^{2 \pi i / p}$ be a primitive $p$-th root of unity. Let $\operatorname{Tr}: \mathbb{F} \rightarrow \mathbb{F}_{p}$ denote the trace operator from $\mathbb{F}$ to $\mathbb{F}_{p}$. The additive characters of $\mathbb{F}$ are given by $\left\{\chi_{a}(x): \mathbb{F} \rightarrow \mathbb{C}: a \in \mathbb{F}\right\}$ defined as

$$
\chi_{a}(x)=\omega^{\operatorname{Tr}(a x)} .
$$

The additive characters of $\mathbb{F}^{t}$ are given by $\chi_{a_{1}, \ldots, a_{t}}\left(x_{1}, \ldots, x_{t}\right)=\Pi_{i=1}^{t} \chi_{a_{i}}\left(x_{i}\right)$ for $a_{1}, \ldots, a_{t} \in \mathbb{F}$. First, we bound the Fourier coefficients of the distribution of $X=\left(X_{1}, \ldots, X_{t}\right)$. The $\left(a_{1}, \ldots, a_{t}\right)$ Fourier coefficient, for all non-zero $\left(a_{1}, \ldots, a_{t}\right)$, is given by

$$
\begin{aligned}
\mathbb{E}\left[\chi_{a_{1}, \ldots, a_{t}}\left(X_{1}, \ldots, X_{t}\right)\right] & =\mathbb{E}\left[\omega^{\operatorname{Tr}\left(\sum_{i=1}^{t} a_{i} X_{i}\right)}\right]=\sum_{b \in \mathbb{F}} \omega^{\operatorname{Tr}(b)} \operatorname{Pr}\left[\sum_{i=1}^{t} a_{i} X_{i}=b\right] \\
& =\sum_{b \in \mathbb{F}} \omega^{\operatorname{Tr}(b)}\left(\underset{X}{\operatorname{Pr}}\left[\sum_{i=1}^{t} a_{i} X_{i}=b\right]-\frac{1}{|\mathbb{F}|}\right),
\end{aligned}
$$

where we used the fact that $\sum_{b \in \mathbb{F}} \omega^{\operatorname{Tr}(b)}=0$. Hence for all non-zero $\left(a_{1}, \ldots, a_{t}\right)$,

$$
\left|\mathbb{E}\left[\chi_{a_{1}, \ldots, a_{t}}\left(X_{1}, \ldots, X_{t}\right)\right]\right| \leq \sum_{b \in \mathbb{F}}\left|\operatorname{Pr}\left[\sum_{i=1}^{t} a_{i} X_{i}=b\right]-\frac{1}{|\mathbb{F}|}\right| \leq 2 \varepsilon \cdot|\mathbb{F}| .
$$

Let $p_{a_{1}, \ldots, a_{t}}=\operatorname{Pr}\left[\left(X_{1}, \ldots, X_{t}\right)=\left(a_{1}, \ldots, a_{t}\right)\right]$. By Parseval's identity,

$$
\sum_{a_{1}, \ldots, a_{t} \in \mathbb{F}}\left(p_{a_{1}, \ldots, a_{t}}-\frac{1}{|\mathbb{F}|}\right)^{2}=\sum_{\left(a_{1}, \ldots, a_{t}\right) \neq 0} \mathbb{E}\left[\chi_{a_{1}, \ldots, a_{t}}\left(X_{1}, \ldots, X_{t}\right)\right]^{2} \leq 4 \varepsilon^{2}|\mathbb{F}|^{t+2},
$$

\section{B Proof of Lemma 6.1}

We will need the following fact.

Fact B.1. Let Dec: $\mathcal{X} \times \mathcal{X} \rightarrow \mathcal{M} \cup\{\perp\}$, and Enc: $\mathcal{M} \rightarrow \mathcal{X} \times \mathcal{X}$ be $\varepsilon$-non-malleable scheme in 2 -split state model for some $\varepsilon<\frac{1}{2}$. For any two messages $m_{0}, m_{1} \in \mathcal{M}$, there exist $x_{1}^{0}, x_{1}^{1}, x_{2} \in \mathcal{X}$ such that

- $\operatorname{Dec}\left(x_{1}^{0}, x_{2}\right)=m_{0}$

- $\operatorname{Dec}\left(x_{1}^{1}, x_{2}\right)=m_{1}$

Proof. By contradiction, let us assume there exists $m_{0}, m_{1} \in \mathcal{M}$ such that $\forall_{x_{2} \in \mathcal{X}} \mid \operatorname{Dec}\left(\mathcal{X}, x_{2}\right) \cap$ $\left\{m_{0}, m_{1}\right\} \mid=1$. Let us define sets $\mathcal{X}_{2}^{0}, \mathcal{X}_{2}^{1}$ as follows

$$
\begin{aligned}
& \mathcal{X}_{2}^{0}=\left\{x_{2} \in \mathcal{X}: \operatorname{Dec}\left(\mathcal{X}, x_{2}\right) \cap\left\{m_{0}, m_{1}\right\}=\left\{m_{0}\right\}\right\} \\
& \mathcal{X}_{2}^{1}=\left\{x_{2} \in \mathcal{X}: \operatorname{Dec}\left(\mathcal{X}, x_{2}\right) \cap\left\{m_{0}, m_{1}\right\}=\left\{m_{1}\right\}\right\}
\end{aligned}
$$

Fix arbitrary $x_{2}^{0} \in \mathcal{X}_{2}^{0}, x_{2}^{1} \in \mathcal{X}_{2}^{1}$, and let

$$
\begin{aligned}
& \mathcal{X}_{1}^{0}=\left\{x \in \mathcal{X} \quad: \operatorname{Dec}\left(x, x_{2}^{0}\right)=m_{0}\right\} \\
& \mathcal{X}_{1}^{1}=\left\{x \in \mathcal{X}: \operatorname{Dec}\left(x, x_{2}^{1}\right)=m_{1}\right\}
\end{aligned}
$$


Consider tampering functions $h_{1}: \mathcal{X} \rightarrow \mathcal{X}$, and $h_{2}: \mathcal{X} \rightarrow \mathcal{X}$ as follows. Let $h_{2}\left(\mathcal{X}_{2}^{0}\right):=x_{2}^{1}$ and $h_{2}\left(\mathcal{X}_{2}^{1}\right):=x_{2}^{0}$, and $h_{2}$ is defined arbitrarily in $\mathcal{X} \backslash\left(\mathcal{X}_{2}^{0} \cup \mathcal{X}_{2}^{1}\right)$. Also, if $\mathcal{X}_{1}^{0} \cap \mathcal{X}_{1}^{1}$ is non-empty, then fix some $x \in \mathcal{X}_{1}^{0} \cap \mathcal{X}_{1}^{1}$, and let $h_{1}(c)=x$ for all $c \in \mathcal{X}$. Otherwise choose arbitrary $x_{1}^{0} \in \mathcal{X}_{1}^{0}, x_{1}^{1} \in \mathcal{X}_{1}^{1}$, and let $h_{1}\left(\mathcal{X}_{1}^{0}\right):=x_{1}^{1}$ and $h_{1}\left(\mathcal{X}_{1}^{1}\right):=x_{1}^{0}$, and $h_{1}$ is defined arbitrarily in $\mathcal{X} \backslash\left(\mathcal{X}_{1}^{0} \cup \mathcal{X}_{1}^{1}\right)$.

Then, we have that Tamper $_{m_{0}}=m_{1}$, and Tamper $_{m_{1}}=m_{0}$ with probability 1 , where Tamper $m_{0}$ and Tamper $_{m_{1}}$ are as in Definition 3.2. This implies that there exists a distribution $D$ over $\mathcal{M} \cup\{\perp\}$ such that $\operatorname{Pr}\left(D=m_{0}\right) \geq 1-\varepsilon$, and $\operatorname{Pr}\left(D=m_{1}\right) \geq 1-\varepsilon$, which is a contradiction.

Lemma 6.1 Let Dec: $\mathcal{X} \times \mathcal{X} \rightarrow \mathcal{M}$, and Enc: $\mathcal{M} \rightarrow \mathcal{X} \times \mathcal{X}$ be $\varepsilon$-non-malleable scheme in 2 -split state model for some $\varepsilon<\frac{1}{2}$. For any pair of messages $m_{0}, m_{1} \in \mathcal{M}$, let $\left(X_{1}^{0}, X_{2}^{0}\right) \leftarrow$ $\operatorname{Enc}\left(m_{0}\right)$, and let $\left(X_{1}^{1}, X_{2}^{1}\right) \leftarrow \operatorname{Enc}\left(m_{1}\right)$. Then $\Delta\left(X_{1}^{0} ; X_{1}^{1}\right) \leq 2 \varepsilon$.

Proof. By contradiction assume that $\Delta\left(X_{1}^{0} ; X_{1}^{1}\right)>2 \varepsilon$. Then there exists distinguisher $\mathcal{A}: \mathcal{X} \rightarrow$ $\{0,1\}$ such that

$$
\operatorname{Pr}\left(\mathcal{A}\left(X_{1}^{0}\right)=1\right)-\operatorname{Pr}\left(\mathcal{A}\left(X_{1}^{1}\right)=1\right)>2 \varepsilon .
$$

By Fact B.1 we have $x_{1}^{0}, x_{1}^{1}, x_{2} \in \mathcal{X}$ such that $\operatorname{Dec}\left(x_{1}^{0}, x_{2}\right)=m_{0}$ and $\operatorname{Dec}\left(x_{1}^{1}, x_{2}\right)=m_{1}$. Now let us choose following tampering functions:

$$
h_{2}(r)=x_{2} \text {, and } h_{1}(\ell)=x_{\mathcal{A}(\ell)} .
$$

Consider Tamper $_{m_{0}}$ and Tamper $_{m_{1}}$ as in Definiton 3.2. By Equation B.2, we have that

$$
\operatorname{Pr}\left[\text { Tamper }_{m_{0}}=m_{1}\right]-\operatorname{Pr}\left[\text { Tamper }_{m_{1}}=m_{1}\right]>2 \varepsilon .
$$

From Definition 3.2 , we have that there exists a distribution $D$ such that

$\mid \operatorname{Pr}\left[\right.$ Tamper $\left._{m_{0}}=m_{1}\right]-\operatorname{Pr}\left(D=m_{1}\right) \mid \leq \varepsilon, \quad$ and $\left.\quad \mid \operatorname{Pr}_{\text {Tamper }_{m_{1}}}=m_{1}\right]-\operatorname{Pr}\left(D=m_{1}\right)-\operatorname{Pr}(D=$ same $) \mid \leq \varepsilon$.

By triangle inequality, this implies,

$$
\operatorname{Pr}\left[\text { Tamper }_{m_{0}}=m_{1}\right]-\operatorname{Pr}\left[\text { Tamper }_{m_{1}}=m_{1}\right]+\operatorname{Pr}(D=\text { same }) \leq 2 \varepsilon,
$$

which contradicts Equation B.3. 